\title{
TOPOLOGICAL QUIVERS AS MULTIPLICITY FREE RELATIONS
}

\author{
BERNDT BRENKEN
}

\begin{abstract}
For a $\mathrm{C}^{*}$-correspondence $\mathscr{E}$ over a $\mathrm{C}^{*}$-algebra $A$ the restricted correspondence $\mathscr{R}(\mathscr{E})$ over the ideal $I=\overline{\langle\mathscr{E}, \mathscr{E}\rangle}$ of $A$ is introduced. The Cuntz-Pimsner algebra $\mathscr{O}_{\mathscr{R}(\mathscr{E})}$ is the unaugmented $\mathrm{C}^{*}$ algebra associated with $\mathscr{E}$. For a topological quiver $G$ an associated multiplicity free quiver, or topological relation, $G^{1}$ is introduced. The Cuntz-Pimsner algebra $\mathscr{O}_{\mathscr{R}(\mathscr{E})}$ of the correspondence $\mathscr{E}$ associated with $G$ is contained in the algebra $\mathscr{O}_{\mathscr{R}(\mathscr{E} 1)}$ for the correspondence $\mathscr{E}^{1}$ associated with $G^{1}$ if the source map for the quiver is proper on an appropriate codomain. The unaugmented Cuntz-Pimsner algebras for $G$ and $G^{1}$ are isomorphic if the left action for the correspondence $\mathscr{R}(\mathscr{E})$ is by compact adjointable maps and if the kernel for the left action is complemented in $I$. There are counter examples if either condition fails.
\end{abstract}

\section{Introduction}

In [2] a topological analogue of a multiplicity free discrete directed graph, called a topological relation, and a procedure associating a $\mathrm{C}^{*}$-correspondence with it, were introduced. Almost simultaneously a detailed study of a $\mathrm{C}^{*}$ correspondence associated with a topological quiver - a topological analogue of a discrete directed graph with multiplicity - was initiated in [14]. In both these approaches a pair of continuous maps was involved, with one of the pair required to be open. Another approach was initiated in [8], with the more restrictive condition that one of these maps was required to be a local homeomorphism. Since $C^{*}$-algebras are naturally formed from $C^{*}$-correspondences ([15]) this provided a method of associating $C^{*}$-algebras with topological analogues of directed graphs. Please refer to [14] for further historical context. In this paper we introduce a multiplicity free relation, analogous to the edge graph of a discrete directed graph, associated with a topological quiver. We show that under a finiteness condition and a boundary condition on the sinks of a quiver that the $\mathrm{C}^{*}$-algebras associated with both the original quiver and its associated multiplicity free quiver are isomorphic.

For a general correspondence $\mathscr{E}$ over a $\mathrm{C}^{*}$-algebra $A$ one can form the Cuntz-Pimsner $\mathrm{C}^{*}$-algebra $\mathscr{O}_{\mathscr{E}}$ associated with $\mathscr{E}$ : it is a universal $\mathrm{C}^{*}$-algebra

Received 20 August 2008. 
for representations of the correspondence $\mathscr{E}$ subject to relations determined by an ideal of $A$. This $C^{*}$-algebra contains an isomorphic copy of the algebra $A$ and can be viewed as the augmented Cuntz-Pimsner $C^{*}$-algebra associated with $\mathscr{E}$ ([15]). In the following we consider another $\mathrm{C}^{*}$-algebra associated with the correspondence, namely the unaugmented Cuntz-Pimsner $\mathrm{C}^{*}$-algebra (originally denoted $\mathcal{O}_{\mathscr{E}}$ in [15]) generated only by the elements in the Hilbert module $\mathscr{E}$. This $\mathrm{C}^{*}$-algebra contains a canonical ideal of the algebra $A$ which is generally proper. For a given $\mathrm{C}^{*}$-correspondence $\mathscr{E}$ over $A$ we introduce a restricted correspondence $\mathscr{R}(\mathscr{E})$ over this ideal of $A$, and show that its universal (augmented) Cuntz-Pimsner $\mathrm{C}^{*}$-algebra $\mathscr{O}_{\mathscr{R}(\mathscr{E})}$ is the unaugmented Cuntz-Pimsner $\mathrm{C}^{*}$-algebra associated with $\mathscr{E}$.

Given a topological quiver $G$ one can form an associated $C^{*}$-correspondence $\mathscr{E}=\mathscr{E}_{G}$. The Cuntz-Pimsner $\mathrm{C}^{*}$-algebra of this correspondence $\mathscr{O}_{\mathscr{E}}$ can be viewed as the $\mathrm{C}^{*}$-algebra of the quiver $G$. We show that if one considers the unaugmented Cuntz-Pimsner $\mathrm{C}^{*}$-algebra $\mathscr{O}_{\mathscr{R}(\mathscr{E})}$ as the $\mathrm{C}^{*}$-algebra of the given quiver, then it is this unaugmented $\mathrm{C}^{*}$-algebra that is appropriate to consider for certain natural properties; namely under certain finiteness conditions it remains unchanged if one replaces the given topological quiver $G$ with an associated multiplicity free topological quiver, or topological relation, $G^{1}$. This yields a canonical isomorphism of $\mathrm{C}^{*}$-algebras associated with differing underlying topological structures. The process of forming $G^{1}$ is defined for any topological quiver $G$. The analogous isomorphism results for arbitrary directed discrete graphs with countable discrete vertex and edge spaces is found in [1].

As a simple and well known illustration of this isomorphism consider the discrete graph defined by a directed edge of multiplicity 2 on a single vertex. The Cuntz-Pimsner $\mathrm{C}^{*}$-algebra $\mathscr{O}_{\mathscr{E}}$ for the associated correspondence $\mathscr{E}=\mathscr{E}_{G}$ is the Cuntz algebra $\mathscr{O}_{2}$ and is the universal $\mathrm{C}^{*}$-algebra generated by two isometries $S_{0}$ and $S_{1}$ with $S_{k}^{*} S_{k}=\sum S_{j} S_{j}^{*}$ for $k=0,1$. The topological relation $G^{1}$ is the associated edge graph, so is the directed graph with two vertices $v_{1}$ and $v_{2}$ with four edges $e_{k}(k \in\{0,1,2,3\})$ with $e_{0}$ a loop on $v_{1}, e_{3}$ a loop on $v_{2}, e_{2}$ an edge from $v_{1}$ to $v_{2}$ and $e_{1}$ an edge from $v_{2}$ to $v_{1}$. The CuntzPimsner $\mathrm{C}^{*}$-algebra $\mathscr{O}_{\mathscr{E} 1}$ for the associated correspondence $\mathscr{E}^{1}=\mathscr{E}_{G^{1}}$ is the universal $\mathrm{C}^{*}$-algebra generated by four partial isometries $T_{k}$ with $T_{0}^{*} T_{0}=$ $T_{1}^{*} T_{1}=T_{0} T_{0}^{*}+T_{2} T_{2}^{*}$ and $T_{2}^{*} T_{2}=T_{3}^{*} T_{3}=T_{1} T_{1}^{*}+T_{3} T_{3}^{*}$. The algebra $*_{-}$ homomorphism $\tau: \mathscr{O}_{\mathscr{E}} \rightarrow \mathscr{O}_{\mathscr{E} 1}$ described on generators by $\tau\left(S_{k}\right)=T_{k}+T_{k+2}$ is an isomorphism, with inverse $\rho$ defined on generators by $\rho\left(T_{0}\right)=S_{0} S_{0} S_{0}^{*}$, $\rho\left(T_{1}\right)=S_{1} S_{0} S_{0}^{*}, \rho\left(T_{2}\right)=S_{0} S_{1} S_{1}^{*}$, and $\rho\left(T_{3}\right)=S_{1} S_{1} S_{1}^{*}$.

One can view the study of topological quivers and their associated universal $\mathrm{C}^{*}$-algebras as extending the study of topological dynamical systems and their associated crossed product $\mathrm{C}^{*}$-algebras, since any continuous map $f: X \rightarrow Y$ 
of locally compact topological spaces gives rise to a canonical (multiplicity free) topological relation ([2], [3]). For example if $f$ is a homeomorphism of a compact space then the Cuntz-Pimsner $\mathrm{C}^{*}$-algebra of the associated topological relation is the usual crossed product $\mathrm{C}^{*}$-algebra ([15], [2]).

It is worth recalling that in [10] it was shown that the $\mathrm{C}^{*}$-algebras of topological graphs, a concept more specialized than that of topological quivers, already contain the $\mathrm{C}^{*}$-algebras appearing in the 'Elliott' classification program of recent years ([16]). The ability to consider multiplicity free topological quivers in certain situations should lead to simplifications in the study of the structure of the associated $\mathrm{C}^{*}$-algebras. For example an application of the results here appears in [4], where under some hypothesis a cross product $\mathrm{C}^{*}$ algebra structure arising from a shift dynamics on a locally compact infinite path space is given for the $\mathrm{C}^{*}$-algebra (of a core subquiver) of a topological quiver. It is anticipated that the associated topological relation $G^{1}$ will be of further use to investigations of $\mathrm{C}^{*}$-algebras of topological quivers $G$.

A summary of the contents follows. Section 1 begins with some preliminary definitions and concepts from the literature, extending where necessary for our purposes some of the current context. For a given correspondence $\mathscr{E}$ over a $\mathrm{C}^{*}$-algebra $A$ we then introduce the restricted correspondence $\mathscr{R}(\mathscr{E})$ over the ideal $\overline{\langle\mathscr{E}, \mathscr{E}\rangle}$ of $A$ and show that the universal Cuntz-Pimsner $\mathrm{C}^{*}$-algebra $\mathscr{O}_{\mathscr{R}(\mathscr{E})}$ of $\mathscr{R}(\mathscr{E})$ is the unaugmented $\mathrm{C}^{*}$-algebra generated by the space $\mathscr{E}$ alone.

For an arbitrary topological quiver $G$ there is an associated correspondence $\mathscr{E}_{G}$, and in Section 2 we consider the restricted correspondence for $G$. The desingularization $G^{1}$ of a topological quiver $G$ is introduced and properties of this multiplicity free quiver and its associated restricted correspondence are determined. A crucial tensor product decomposition for the restricted correspondence $\mathscr{R}\left(\mathscr{E}^{1}\right)$ associated with $G^{1}$ is established. This decomposition should provide a general approach to defining a desingularization procedure for a general $\mathrm{C}^{*}$-correspondences.

A description of the proper part of a $\mathrm{C}^{*}$-algebra homomorphism is introduced in the following section and an elementary property of the induced algebra map for a map of topological spaces is given. This leads to the idea of a proper quiver, which extends that of a proper map if the quiver arises from a continuous map $f: X \rightarrow Y$. For such a quiver $G$ there is a natural coisometric morphism from the restricted correspondence $\mathscr{R}(\mathscr{E})$ for the quiver $G$ to the restricted correspondence $\mathscr{R}\left(\mathscr{E}^{1}\right)$ for the associated multiplicity free quiver $G^{1}$ yielding an injection of $\mathscr{O}_{\mathscr{R}(\mathscr{E})}$ into $\mathscr{O}_{\mathscr{R}(\mathscr{E} 1)}$.

Section 4 establishes further conditions on the quiver $G$ under which this injection is an isomorphism of $\mathrm{C}^{*}$-algebras. One condition, termed range finite, involves the left action of the restricted correspondence acting by compact adjointable operators. For many quivers, including discrete directed graphs and 
the topological graphs considered by Katsura, this is automatically satisfied by the previous condition of being proper, so entails nothing new. A second condition involves a topological restriction on how the kernel of this left action sits inside the range ideal, namely the sinks of the quiver are a closed, hence clopen, subset of the range subspace of the vertex space. This later condition is trivially satisfied, for example, if this subspace is discrete, or if $G$ has no nontrivial sinks. The section concludes with some examples illustrating nonisomorphism if various hypothesis are not satisfied.

\section{Notation}

For $A, B$, subsets of a set $Y, A-B$ is the set of those points in $A$ and not in $B$. If $A$ is a subset of a topological space $Y$ then the closure of $A$ is denoted by $\mathrm{Cl}_{Y} A$, or if there is no ambiguity by $\bar{A}$, the complement of $A$ is denoted $Y-A$, or by $A^{c}$ if the context is clear, while $\partial_{Y} A=\bar{A} \cap(\overline{Y-A})$ is the boundary of $A$. The interior of $A$ is $\operatorname{Int}_{Y} A$. The *-algebra of continuous functions on $Y$ is $C(Y)$, and the *-algebra of bounded continuous functions on $Y$ is $C_{b}(Y)$. If $Y$ is locally compact Hausdorff $C_{c}(Y)$ is the algebra of continuous functions with compact support. Its closure in the uniform sup norm, \|\|$_{\infty}$, is the algebra of continuous functions that vanish at infinity, $C_{0}(Y)$. For $K$ a compact set in $Y$ the subalgebra of functions in $C_{c}(Y)$ with support in $K$ is $C_{K}(Y)$. A Radon measure on $Y$ is a positive linear functional on $C_{c}(Y)$, so is continuous when viewed on the subalgebra $C_{K}(Y)$ for $K$ compact in $Y$. The supports of a function $f$ or of a measure $\lambda$ are denoted $\operatorname{supp}(f)$ and $\operatorname{supp}(\lambda)$ respectively. If $f: Y \rightarrow Z$ is a continuous map of topological spaces then $\operatorname{dom}(f)$ and $\operatorname{ran}(f)$ denote the domain and range respectively of $f$, and the dual map $f^{\sharp}: C(Z) \rightarrow C(Y)$ is given by $f^{\sharp}(h)=h \circ f$. Note that if $i: A \rightarrow Y$ is the inclusion map of a closed subspace $A$ of topological space $Y$ then $i^{\#}: C_{c}(Y) \rightarrow C_{c}(A)$ is the restriction map. By an ideal of a $C^{*}$-algebra $A$ we shall mean a closed two sided ideal, and if $B$ is a subset of a $C^{*}$-algebra $A$ then $\mathscr{I}(B)$ denotes the ideal of $A$ generated by $B$. For an ideal $J$ of $A, J^{\perp}$ denotes the ideal $\{a \in A \mid a b=0,(b \in J)\}$. The multiplier algebra of the $\mathrm{C}^{*}$-algebra $A$ is denoted $M(A)$. As usual $\mathscr{K}$ denotes the $\mathrm{C}^{*}$-algebra of compact operators on a separable Hilbert space and $\mathscr{T}$ the Toeplitz algebra, so the $\mathrm{C}^{*}$-algebra generated by an isometry.

\section{The unaugmented $C^{*}$-algebra of a correspondence}

For results and conventions on $\mathrm{C}^{*}$-modules we follow Lance [11]; so if $A$ is a $\mathrm{C}^{*}$-algebra a Hilbert $A$-module $\mathscr{E}$ is a Banach space which is a right $A$-module with an $A$-valued inner product $\langle,\rangle_{A}$, denoted $\langle$,$\rangle if the context is clear. The$ norm on $\mathscr{E}$ is given by $\|x\|^{2}=\|\langle x, x\rangle\|,(x \in \mathscr{E}) ; \mathscr{L}(\mathscr{E})$ denotes the $\mathrm{C}^{*}$ algebra of adjointable operators on $\mathscr{E}$ while $\mathscr{K}(\mathscr{E})$, in analogy with the case 
when $A$ is the complex numbers, is the closed two-sided ideal of 'compact' operators $\overline{\operatorname{span}}\left\{\theta_{x, y}^{\mathscr{E}} \mid x, y \in \mathscr{E}\right\}$ where $\theta_{x, y}^{\mathscr{E}}(z)=x\langle y, z\rangle,(z \in \mathscr{E})$. If $\mathscr{E}$ is a Hilbert $A$-module the linear span of $\{\langle x, y\rangle \mid x, y \in \mathscr{E}\}$, denoted $\langle\mathscr{E}, \mathscr{E}\rangle$, has closure a two-sided ideal of $A$. Note that $\mathscr{E}\langle\mathscr{E}, \mathscr{E}\rangle$ is dense in $\mathscr{E}$ ([11]). The Hilbert module $\mathscr{E}$ is called full if $\langle\mathscr{E}, \mathscr{E}\rangle$ is dense in $A$. If $A$ is a $\mathrm{C}^{*}$-algebra then $A_{A}$ refers to the Hilbert module $A$ over itself, where $\langle a, b\rangle=a^{*} b$ for $a, b \in A$.

Definition 1.1. If $A, B$ are $C^{*}$-algebras then a $C^{*}$-correspondence $\mathscr{E}$ from $A$ to $B$ is a right Hilbert $B$-module $\mathscr{E}$ together with a left action of $A$ on $\mathscr{E}$ given by a $*$-homomorphism $\phi_{A}: A \rightarrow \mathscr{L}(\mathscr{E}), a \cdot x=\phi_{A}(a) x$, for $a \in A$, $x \in \mathscr{E}$. Just $\phi$ is used if the context is clear. When convenient the notation ${ }_{A} \mathscr{E}_{B}$ is used to denote a $C^{*}$-correspondence from $A$ to $B$. If $A=B$ refer to $\mathscr{E}$ as a correspondence over $A$.

The identity correspondence $A$ over $A$ is $A$ viewed as a Hilbert module over itself with the left action given by left multiplication.

If $\mathscr{E}$ and $\mathscr{F}$ are $C^{*}$-correspondence over $C^{*}$-algebras $A$ and $B$ respectively then a morphism $(T, \pi): \mathscr{E} \rightarrow \mathscr{F}$ is a $*$-homomorphism $\pi: A \rightarrow B$ and a linear map $T: \mathscr{E} \rightarrow \mathscr{F}$ with

1. $\pi\left(\langle e, f\rangle_{A}\right)=\langle T(e), T(f)\rangle_{B}$

2. $T\left(\phi_{A}(a) e\right)=\phi_{B}(\pi(a)) T(e)$

3. $T(e) \pi(a)=T(e a)$

for all $e, f \in \mathscr{E}, a \in A$.

Condition 1 ensures that $T$ is a continuous linear map of bound 1 , and if $\pi$ restricted to the ideal $\overline{\langle\mathscr{E}, \mathscr{E}\rangle}$ of $A$ is injective then $T$ must be an isometry of Hilbert modules, so injective. Thus a morphism $(T, \pi): \mathscr{E} \rightarrow \mathscr{F}$ is an isomorphism if $T: \mathscr{E} \rightarrow \mathscr{F}$ is a surjection and $\pi$ restricted to the ideal $\overline{\langle\mathscr{E}, \mathscr{E}\rangle}$ of $A$ is injective. Evidently the composition of two morphisms is a morphism. A representation of $\mathscr{E}$ in a $C^{*}$-algebra $B$ is a correspondence morphism $(T, \pi)$ : $\mathscr{E} \rightarrow B$ where $B$ is viewed as the identity correspondence.

It is still the case for a morphism of correspondences $(T, \pi): \mathscr{E} \rightarrow \mathscr{F}$, as noted in [14] in the case that $(T, \pi)$ is a representation in a $\mathrm{C}^{*}$-algebra, that the third condition is a consequence of the first, since $\|T(e) \pi(a)-T(e a)\|^{2}$ is the norm in $B$ of the element $\langle T(e) \pi(a)-T(e a), T(e) \pi(a)-T(e a)\rangle_{B}$. The first condition along with properties of the $B$-valued inner product ensure however that this inner product is equal to 0 .

If $(T, \pi): \mathscr{E} \rightarrow B$ is a representation of $\mathscr{E}$ in a $C^{*}$-algebra $B$ the $\mathrm{C}^{*}$ subalgebra of $B$ generated by $T(\mathscr{E}) \cup \pi(A)$ is denoted $C^{*}(T, \pi)$. If $\rho: B \rightarrow C$ is a *homomorphism of $C^{*}$-algebras then $(\rho \circ T, \rho \circ \pi)$ is a representation of $\mathscr{E}$ in $C$, denoted $\rho \circ(T, \pi)$. A morphism of correspondences $(T, \pi)$ from $\mathscr{E}$ to 
$\mathscr{F}$ yields a $*$-homomorphism $\Psi_{T}: \mathscr{K}(\mathscr{E}) \rightarrow \mathscr{K}(\mathscr{F})$ by $\Psi_{T}\left(\theta_{x, y}\right)=\theta_{T(x), T(y)}$ for $x, y \in \mathscr{E}([6])$, and if $(S, \sigma): \mathscr{D} \rightarrow \mathscr{E},(T, \pi): \mathscr{E} \rightarrow \mathscr{F}$ are morphisms of $\mathrm{C}^{*}$-correspondences then $\Psi_{T} \circ \Psi_{S}=\Psi_{T \circ S}$. For $B$ a C*-algebra, using the identification of $\mathscr{K}(B)$ with $B$, a representation $(T, \pi)$ of $\mathscr{E}$ in a $C^{*}$-algebra $B$ yields a *-homomorphism $\Psi_{T}: \mathscr{K}(\mathscr{E}) \rightarrow B$ given by $\theta_{x, y} \rightarrow T(x) T^{*}(y)$. As noted in [3] the argument of Lemma 2.2 [6] showing that $\Psi_{T}$ is injective if $\pi$ is injective also serves to show that $\Psi_{T}$ is injective if only the restriction of $\pi$ to the ideal $\overline{\langle\mathscr{E}, \mathscr{E}\rangle}$ of $A$ is injective.

Proposition 1.2. Let $\mathscr{E}$ be a $C^{*}$-correspondence over A, $\mathscr{F}$ a $C^{*}$-correspondence over $B$ and $(T, \pi): \mathscr{E} \rightarrow \mathscr{F}$ a morphism. If $\Psi_{T}: \mathscr{K}(\mathscr{E}) \rightarrow \mathscr{K}(\mathscr{F})$ is the *-homomorphism defined by $(T, \pi)$ then

1. $\phi_{B}(\pi(a)) \Psi_{T}(\theta)=\Psi_{T}\left(\phi_{A}(a) \theta\right)$ and $\Psi_{T}(\theta) \phi_{B}(\pi(a))=\Psi_{T}\left(\theta \phi_{A}(a)\right)$

2. $\Psi_{T}(\theta) T(e)=T(\theta(e))$

for all $a \in A, \theta \in \mathscr{K}(\mathscr{E}), e \in \mathscr{E}$.

Proof. To check these conditions it is enough to show them for $\theta=\theta_{x, y}$, $x, y \in \mathscr{E}$. For $z \in \mathscr{F}$ we have

$$
\begin{aligned}
\phi_{B}(\pi(a)) \Psi_{T}\left(\theta_{x, y}\right)(z) & =\phi_{B}(\pi(a))\left(\theta_{T x, T y}(z)\right)=\phi_{B}(\pi(a))(T x\langle T y, z\rangle) \\
& =T\left(\phi_{A}(\pi(a) x)\langle T y, z\rangle\right)=\Psi_{T}\left(\theta_{\phi_{A(a) x, y}}\right)(z) \\
& =\Psi_{T}\left(\phi_{A}(a) \theta_{x, y}\right)(z) .
\end{aligned}
$$

The second equality follows from the first by taking adjoints and noting that $\Psi_{T}, \pi, \phi_{A}$, and $\phi_{B}$ are *-homomorphisms.

The left hand side of the second condition is $\theta_{T x, T y} T(e)=T(x)\langle T y, T e\rangle_{B}$ $=T(x) \pi\left(\langle y, e\rangle_{A}\right)=T\left(x\langle y, e\rangle_{A}\right)=T\left(\theta_{x, y}(e)\right)$ from Definition 1.1.

For $\mathscr{E}$ a $\mathrm{C}^{*}$-correspondence over $A$ denote the ideal $\phi^{-1}(\mathscr{K}(\mathscr{E}))$ of $A$ by $J(\mathscr{E})$, and the ideal $J(\mathscr{E}) \cap(\operatorname{ker} \phi)^{\perp}$ by $J_{\mathscr{E}}$.

Definition 1.3. If $\mathscr{E}$ and $\mathscr{F}$ are $\mathrm{C}^{*}$-correspondence over $\mathrm{C}^{*}$-algebras $A$ and $B$ respectively and $K$ an ideal in $J(\mathscr{E})$, a morphism $(T, \pi): \mathscr{E} \rightarrow \mathscr{F}$ is coisometric on $K$ if $\Psi_{T}\left(\phi_{A}(a)\right)=\phi_{B}(\pi(a))$ for all $a \in K$. A morphism is coisometric if $K=J(\mathscr{E})$.

Note that since $\Psi_{T}: \mathscr{K}(\mathscr{E}) \rightarrow \mathscr{K}(\mathscr{F})$ this equality can only occur if $\phi_{B}(\pi(a)) \in \mathscr{K}(\mathscr{F})$, i.e., if $\pi(K) \subseteq J(\mathscr{F})$.

Given a $C^{*}$-correspondence $\mathscr{E}$ over $A$ and $K$ an ideal in $J(\mathscr{E})$ there is a representation $\left(T_{\mathscr{E}}, \pi_{\mathscr{E}}\right)$ of $\mathscr{E}$ in a $\mathrm{C}^{*}$-algebra which is coisometric on $K$ and universal among all such representations ([5]), in the sense that if $(T, \pi)$ is a representation of $\mathscr{E}$ in a $\mathrm{C}^{*}$-algebra $B$ which is coisometric on $K$ then there is a ${ }^{*}$-homomorphism $\rho: C^{*}\left(T_{\mathscr{E}}, \pi_{\mathscr{E}}\right) \rightarrow B$ with $(T, \pi)=\rho \circ\left(T_{\mathscr{E}}, \pi_{\mathscr{E}}\right)$. 
The $C^{*}$-algebra $C^{*}\left(T_{\mathscr{E}}, \pi_{\mathscr{E}}\right)$ is called the relative Cuntz-Pimsner algebra of $\mathscr{E}$ determined by $K$ and denoted $\mathcal{O}(K, \mathscr{E})$. When $K=0$ the $\mathrm{C}^{*}$-algebra $\mathcal{O}(K, \mathscr{E})$ is denoted $\mathscr{T}(\mathscr{E})$ and called the universal Toeplitz $\mathrm{C}^{*}$-algebra for $\mathscr{E}$. The $\mathrm{C}^{*}$ algebra $\mathscr{O}\left(J_{\mathscr{E}}, \mathscr{E}\right)$ is denoted $\mathscr{O}_{\mathscr{E}}$.

A representation $(T, \pi): \mathscr{E} \rightarrow B$ of a correspondence $\mathscr{E}$ over $A$ in a $C^{*}$-algebra $B$ is said to admit a gauge action $\gamma$ if $\gamma: \mathrm{T} \rightarrow \operatorname{Aut} C^{*}(T, \pi)$ is a homomorphism with $\gamma_{t}(T(e))=t T(e)$ for all $e \in \mathscr{E}$ and $\gamma_{t}(\pi(a))=$ $\pi(a)$ for all $a \in A,(t \in \mathrm{T})$. Since the *-homomorphism $\Psi_{T}$ is defined by mapping $\theta_{x, y} \rightarrow T(x) T^{*}(y) \in C^{*}(T, \pi)$ we have that $\gamma_{t}\left(\Psi_{T}(\theta)\right)=\Psi_{T}(\theta)$ for $\theta \in \mathscr{K}(\mathscr{E})$ and $t \in \mathrm{T}$. The universal representation $\left(T_{\mathscr{E}}, \pi_{\mathscr{E}}\right)$ of $\mathscr{E}$ which is coisometric on $K$ admits a gauge action, called the canonical gauge action, on $\mathcal{O}(K, \mathscr{E})$ : this follows from the universal property of $\mathcal{O}(K, \mathscr{E})$. The GaugeInvariant Uniqueness Theorem (cf. [12]) states that if $(T, \pi): \mathscr{E} \rightarrow B$ is a representation of a correspondence $\mathscr{E}$ in a $C^{*}$-algebra $B$ coisometric on $J_{\mathscr{E}}$ then the induced $*$-homomorphism $\rho: \mathscr{O}_{\mathscr{E}} \rightarrow C^{*}(T, \pi)$ is an isomorphism if and only if $\pi$ is injective and $(T, \pi)$ admits a gauge action.

Proposition 1.4. Let $\mathscr{E}$ be a $C^{*}$-correspondence over $A$ and $\mathscr{F}$ a $C^{*}$ correspondence over $B$. Let $\left(T_{\mathscr{E}}, \pi_{\mathscr{E}}\right)$ be a universal representation of $\mathscr{E}$ in $\mathcal{O}(K, \mathscr{E})$ coisometric on an ideal $K$ in $J(\mathscr{E})$ and $(S, \sigma)$ a representation of $\mathscr{F}$ in a $C^{*}$-algebra $C$ coisometric on an ideal $L$ in $J(\mathscr{F})$. If $(T, \pi): \mathscr{E} \rightarrow$ $\mathscr{F}$ is a morphism coisometric on $K$ with $\pi(K) \subseteq L$ there is a unique $*_{-}$ homomorphism $\rho: \mathcal{O}(K, \mathscr{E}) \rightarrow C$ with $\rho \circ\left(T_{\mathscr{E}}, \pi_{\mathscr{E}}\right)=(S, \sigma) \circ(T, \pi)$.

Proof. It is enough to show that the morphism $(S, \sigma) \circ(T, \pi)=(S \circ T, \sigma \circ$ $\pi)$ is a representation of $\mathscr{E}$ in $C$ coisometric on $K$. However $\Psi_{S \circ T}\left(\phi_{A}(a)\right)=$ $\Psi_{S} \Psi_{T}\left(\phi_{A}(a)\right)=\Psi_{S} \phi_{B}(\pi(a))=\sigma(\pi(a)$ for $a \in K$, the later equality following from noting that $\pi(a) \in L$ and that $(S, \sigma)$ is coisometric on $L$.

Note that the condition $\pi(K) \subseteq L$ is automatically satisfied when $L$ is the ideal $J(\mathscr{F})$. The following is basically a version of the Gauge-Invariant Uniqueness Theorem.

Corollary 1.5. Let $\mathscr{E}$ be a $C^{*}$-correspondence over $A$ and $\mathscr{F}$ a $C^{*}$ correspondence over $B$. Let $\left(T_{\mathscr{E}}, \pi_{\mathscr{E}}\right)$ be a universal representation of $\mathscr{E}$ in $\mathcal{O}_{\mathscr{E}}$ and $\left(T_{\mathscr{F}}, \pi_{\mathscr{F}}\right)$ a universal representation of $\mathscr{F}$ in $\mathscr{O}_{\mathscr{F}}$. If $(T, \pi): \mathscr{E} \rightarrow \mathscr{F}$ is a morphism coisometric on $J_{\mathscr{E}}$ with $\pi\left(J_{\mathscr{E}}\right) \subseteq J_{\mathscr{F}}$ and $\pi$ injective on A then there is a unique injection $\rho: \mathscr{O}_{\mathscr{E}} \rightarrow \mathscr{O}_{\mathscr{F}}$ with $\rho \circ\left(T_{\mathscr{E}}, \pi_{\mathscr{E}}\right)=\left(T_{\mathscr{F}}, \pi_{\mathscr{F}}\right) \circ(T, \pi)$.

Proof. Since $\left(T_{\mathscr{F}}, \pi_{\mathscr{F}}\right)$ admits a gauge action $\gamma$ it is immediate that the representation $\left(T_{\mathscr{F}}, \pi_{\mathscr{F}}\right) \circ(T, \pi)$ satisfies the conditions for a gauge action. Thus the sub $C^{*}$-algebra $C^{*}\left(T_{\mathscr{E}} \circ T, \pi_{\mathscr{E}} \circ \pi\right)$ is invariant under $\gamma$ and the restriction of $\gamma$ is a gauge action for $\left(T_{\mathscr{F}}, \pi_{\mathscr{F}}\right) \circ(T, \pi)$. Since both $\pi_{\mathscr{F}}$ and $\pi$ 
are injective the Gauge-Invariant Uniqueness Theorem implies that the induced *-homomorphism $\rho$ is injective.

Definition 1.6. For a given $\mathrm{C}^{*}$-correspondence $\mathscr{E}$ over $A$ the restricted correspondence $\mathscr{R}(\mathscr{E})$ is the $\mathrm{C}^{*}$-correspondence ${ }_{I} \mathscr{E} I$ over the ideal $I=\overline{\langle\mathscr{E}, \mathscr{E}\rangle}$ of $A$ where the space $\mathscr{E}$ remains unchanged, the original $A$-valued inner product is viewed as having values in the ideal $I$, and the *-homomorphism $\phi_{I}: I \rightarrow$ $\mathscr{L}(\mathscr{E})$ defining the left action of the correspondence is given by $\phi \circ i$, with $i: I \rightarrow A$ the inclusion.

Remark 1.7. For a given $\mathrm{C}^{*}$-correspondence $\mathscr{E}$ over $A$ it is clear that the $\mathrm{C}^{*}$-algebra of adjointable operators $\mathscr{L}(\mathscr{E})$, respectively its $\mathrm{C}^{*}$-ideal $\mathscr{K}(\mathscr{E})$, on the Hilbert $A$-module $\mathscr{E}$ is identical to the $\mathrm{C}^{*}$-algebra $\mathscr{L}(\mathscr{R}(\mathscr{E}))$, respectively $\mathscr{K}(\mathscr{R}(\mathscr{E}))$, of operators on the Hilbert $I$-module $\mathscr{R}(\mathscr{E})$.

Proposition 1.8. If $\mathscr{E}$ is a $C^{*}$-correspondence over a $C^{*}$-algebra $A$ and $\mathscr{R}(\mathscr{E})$ is the restricted correspondence over the $C^{*}$-algebra $I=\overline{\langle\mathscr{E}, \mathscr{E}\rangle}$ then $J(\mathscr{E}) \cap I=J(\mathscr{R}(\mathscr{E}))$ and $J_{\mathscr{E}} \cap I=J_{\mathscr{R}(\mathscr{E})}$.

Proof. The ideal $J_{\mathscr{E}} \cap I=\phi^{-1}(\mathscr{K}(\mathscr{E})) \cap(\operatorname{ker} \phi)^{\perp} \cap I$ is contained in $\phi_{I}^{-1}(\mathscr{K}(\mathscr{E})) \cap\left(\operatorname{ker} \phi_{I}\right)^{\perp}=J_{\mathscr{R}(\mathscr{E})}$ since $\phi^{-1}(\mathscr{K}(\mathscr{E}))=\phi_{I}^{-1}(\mathscr{K}(\mathscr{E})) \cap I$, i.e.; $J(\mathscr{E}) \cap I=J(\mathscr{R}(\mathscr{E}))$. Suppose $a \in J_{\mathscr{R}(\mathscr{E})}$. We need to show that $a x=0$ for $x \in \operatorname{ker} \phi$. However if $e_{\lambda}$ is an approximate unit for $I$ then $e_{\lambda} x \in I \cap \operatorname{ker} \phi=$ $\operatorname{ker} \phi_{I}$ so $a\left(e_{\lambda} x\right)=0$. Taking limits we have $a x=0$.

For the universal representation $(T, \pi)$ of $\mathscr{E}$ coisometric on $J_{\mathscr{E}}$ in the $\mathrm{C}^{*}$ algebra $\mathscr{O}_{\mathscr{E}}$ consider the $\mathrm{C}^{*}$-subalgebra $C^{*}(T(\mathscr{E}))$ of $\mathscr{O}_{\mathscr{E}}$ generated by the subspace $T(\mathscr{E})$.

Proposition 1.9. Let $(T, \pi): \mathscr{E} \rightarrow \mathcal{O}_{\mathscr{E}}$ be the universal representation of a $C^{*}$-correspondence $\mathscr{E}$ over A coisometric on $J_{\mathscr{E}}$. Then the $C^{*}$-subalgebra $C^{*}(T(\mathscr{E}))$ of $\mathscr{O}_{\mathscr{E}}$ is a gauge invariant ideal, and is equal to the $C^{*}$-subalgebra generated by $T(\mathscr{E}) \cup \pi(\overline{(\mathscr{E}, \mathscr{E}\rangle})$.

Proof. Noting that $\pi(a) T(e)=T(\phi(a) e) \subseteq T(\mathscr{E})$ and $T(e) \pi(a)=$ $T(e a) \in T(\mathscr{E})$ for $a \in A, e \in \mathscr{E}$, and that $\pi$ is a *homomorphism it follows that $C^{*}(T(\mathscr{E}))$ is an ideal of $\mathscr{O}_{\mathscr{E}}$. If $\gamma: \mathrm{T} \rightarrow \operatorname{Aut}\left(\mathscr{O}_{\mathscr{E}}\right)$ is the canonical gauge action for the representation $(T, \pi)$ then $\gamma_{t}(T(e))=t T(e) \in C^{*}(T(\mathscr{E}))$ for $e \in \mathscr{E}, t \in \mathrm{T}$, so since $\gamma_{t}$ is a $*$-homomorphism the ideal is gauge invariant. Also, for $e, f \in \mathscr{E}, \pi(\langle e, f\rangle)=T^{*}(e) T(f) \in C^{*}(T(\mathscr{E}))$, so $\pi(\overline{\langle\mathscr{E}, \mathscr{E}\rangle}) \subseteq$ $C^{*}(T(\mathscr{E}))$.

Theorem 1.10. The universal Cuntz-Pimsner $C^{*}$-algebra $\mathscr{O}\left(J_{\mathscr{R}(\mathscr{E})}, \mathscr{R}(\mathscr{E})\right)$ $=\mathcal{O}_{\mathscr{R}(\mathscr{E})}$ associated with the correspondence $\mathscr{R}(\mathscr{E})$ is isomorphic to the $C^{*}$ subalgebra $C^{*}(T(\mathscr{E}))$, generated by the subspace $T(\mathscr{E})$, of the Cuntz-Pimsner $C^{*}$-algebra $\mathcal{O}_{\mathscr{E}}$. 
Proof. Let $(T, \pi)$ be a universal representation of $\mathscr{E}$ coisometric on $J_{\mathscr{E}}$ and $(S, \sigma)$ a universal representation of $\mathscr{R}(\mathscr{E})$ coisometric on $J_{\mathscr{R}(\mathscr{E})}$. The representation $\pi$ is injective, so $\pi_{\mid I}$ is also and $\left(T, \pi_{\mid I}\right)$ is a covariant representation of $\mathscr{R}(\mathscr{E})$ which is coisometric on $J_{\mathscr{E}} \cap I$, which is $J_{\mathscr{R}(\mathscr{E})}$ by Proposition 1.8. The universal property applied to $(S, \sigma)$ yields a $*$-homomorphism $\rho: \mathscr{O}_{\mathscr{R}(\mathscr{E})} \rightarrow \mathcal{O}_{\mathscr{E}}$ with $\rho \circ(S, \sigma)=\left(T, \pi_{\mid I}\right)$. Since the $\mathrm{C}^{*}$-algebra generated by $T(\mathscr{E}) \cup \pi(I)$ is $C^{*}(T(\mathscr{E}))$, and the canonical gauge action for $(T, \pi)$ is a gauge action for the coisometric representation $\left(T, \pi_{\mid I}\right)$ of $\mathscr{R}(\mathscr{E})$, the gauge invariant uniqueness theorem implies that $\rho$ is an isomorphism.

Thus for $\mathscr{E}$ a correspondence over a $\mathrm{C}^{*}$-algebra $A$ and $I$ the ideal $\overline{\langle\mathscr{E}, \mathscr{E}\rangle}$ of $A$, the Cuntz-Pimsner $\mathrm{C}^{*}$-algebra $\mathscr{O}_{\mathscr{R}(\mathscr{E})}$ is the unaugmented universal $\mathrm{C}^{*}$ algebra associated with $\mathscr{E}$.

\section{Desingularization of a topological quiver}

Following [14], $G=(X, E, r, s, \lambda)$ is a topological quiver when $X, E$ are a pair of second countable locally compact Hausdorff spaces, $r: E \rightarrow X$ and $s: E \rightarrow X$ a pair of continuous maps (the range and source maps) with $r$ open, and $\lambda$ a family $\left\{\lambda_{x} \mid x \in X\right\}$ of Radon measures on $E$ with

(1) $\operatorname{supp}\left(\lambda_{x}\right)=r^{-1}(x),(x \in X)$

(2) $x \rightarrow \lambda_{x}(f)=\int_{E} f(\alpha) d \lambda_{x}(\alpha) \in C_{c}(X)$ for $f \in C_{c}(E)$.

A topological quiver $G$ defines a $\mathrm{C}^{*}$-correspondence $\mathscr{E}$ (or $\mathscr{E}_{G}$ ) over the $\mathrm{C}^{*}$ algebra $A=C_{0}(X)$ as follows: for $g, h \in C_{c}(E)$ an $A$-valued inner product given by

$$
\langle g, h\rangle(x)=\int_{r^{-1}(x)} \overline{g(\alpha)} h(\alpha) d \lambda_{x}(\alpha), \quad(x \in X),
$$

defines a norm, $\|f\|^{2}=\left\|\lambda_{x}\left(|f|^{2}\right)\right\|_{\infty}$, on $C_{c}(E)$ with completion $\mathscr{E}$, a Hilbert module over $C_{0}(X)$. The left action arising from a ${ }^{*}$-homomorphism $\phi: A \rightarrow$ $\mathscr{L}(\mathscr{E})$ and the right action of $A$ on an element $h$ of $C_{c}(E)$ are given by

$$
\begin{aligned}
h \cdot a & =h\left(r^{\sharp}(a)\right) \\
\phi(a) h & =\left(s^{\sharp}(a)\right) h,
\end{aligned}
$$

for $a \in A$.

In [14], $X_{\text {sink }}$ denotes the open set of $X$ with $\operatorname{ker} \phi \cong C_{0}\left(X_{\text {sink }}\right)$ and it is shown that $X_{\text {sink }}=X-\overline{s(E)}$, or equivalently that $\overline{s(E)}=\{x \in X \mid f(x)=$ $0,(f \in \operatorname{ker} \phi)\}$, so $\operatorname{ker} \phi=\left\{f \in C_{0}(X)|f| \overline{s(E)} \equiv 0\right\}$. The open subset $X_{\text {fin }}$ of finite emitters of $X$ is defined by $C_{0}\left(X_{\text {fin }}\right) \cong \phi^{-1}(\mathscr{K}(\mathscr{E}))=J(\mathscr{E})$, and it is shown that $X_{\text {fin }}=\left\{x \in X \mid \exists\right.$ neighbourhood $N_{x}$ of $x$ with $\overline{N_{x}}$ and $s^{-1}\left(\overline{N_{x}}\right)$ 
compact with $\left.r\right|_{s^{-1}\left(N_{x}\right)}$ a local homeomorphism\}. Since $(\operatorname{ker} \phi)^{\perp}=C_{0}(X-$ $\left.\overline{X_{\text {sink }}}\right)=C_{0}(\operatorname{Int}(\overline{s(E)}))$ it follows ([14]) that $J_{\mathscr{E}}=C_{0}\left(X_{\text {fin }} \cap \operatorname{Int}(\overline{s(E)})\right)$. If $G$ is a discrete directed graph then $X_{\text {fin }}=X_{\text {fin }} \cap \operatorname{Int}(\overline{s(E)})$ and consists of the vertices of the graph which emit a finite number of edges.

In [3] it is shown that the ideal $I=\overline{\langle\mathscr{E}, \mathscr{E}\rangle}$ of $A$ is $C_{0}(r(E))=\{f \in A \mid$ $f \equiv 0$ on $X \backslash r(E)\}$. Thus, the restricted $\mathrm{C}^{*}$-correspondence $\mathscr{R}(\mathscr{E})$ over the ideal $I=C_{0}(r(E))$ has $\operatorname{ker}\left(\phi_{I}\right)=I \cap \operatorname{ker}(\phi)=C_{0}(r(E)) \cap C_{0}\left(X_{\text {sink }}\right)=$ $C_{0}\left(F_{G}\right)$ where $F_{G}$ denotes the open set $r(E) \cap X_{\text {sink }}=r(E)-\overline{s(E)}$ of sinks that are not isolated in $X$. When the context is clear, $F$ will denote this open subset of $X$ naturally associated with $G$. The ideal $J(\mathscr{R}(\mathscr{E}))=C_{0}\left(X_{\text {fin }} \cap r(E)\right)$ and the ideal $J_{\mathscr{R}(\mathscr{E})}=C_{0}\left(\left(X_{\text {fin }} \cap r(E)\right)-\bar{F}\right)=C_{0}\left(X_{\text {fin }} \cap r(E) \cap \operatorname{Int}(\overline{s(E)})\right)$.

Remark 2.1. Note for a fixed compact set $K$ in $E$ that by definition $\lambda_{x}$ is a bounded linear functional on the Banach space $C_{K}(E)$ for each $x \in X$. Since an element of $C_{c}(X)$ is bounded, the second quiver condition implies that for each $l \in C_{K}(E)$ there is a constant $N_{l}$ such that $\left\|\lambda_{x}(l)\right\|_{\infty} \leq N_{l}$, $(x \in K)$. By the Uniform Boundedness Principle, there is an $N=N_{K}$ so that $\left\|\lambda_{x}(l)\right\|_{\infty} \leq N_{K}\|l\|_{\infty}$ for $l \in C_{K}(E)$ and $x \in X$. Thus a norm dense subset of the Banach space $C_{K}(E)$ is also dense in $C_{K}(E)$ when equipped with the Hilbert $A$-module norm.

Given a topological quiver $G=(X, E, r, s, \lambda)$ we define what we show is a new topological quiver, in fact a topological relation ([2]) $G^{1}=\left(X^{1}, E^{1}, r_{1}\right.$, $\left.s_{1}, \lambda^{1}\right)$ or multiplicity 1 topological quiver associated with $G$. With $F=F_{G}=$ $r(E)-\overline{s(E)}$ as above, the closure of $F$ in $r(E), \mathrm{Cl}_{r(E)}(F)$, is $\bar{F} \cap r(E)$. Since this is the intersection of a closed and open subset in a locally compact space, it is locally compact. We denote this by $\bar{F}$ throughout since that is the only closure of $F$ we consider. Define $X^{1}$ as the disjoint union or coproduct of the topological spaces $E$ and $\bar{F}$, so $X^{1}=E \sqcup \bar{F}$. To define $E^{1}$ first consider the disjoint union of $s^{-1}(r(E))$ and $\bar{F}$, a locally compact subset of $X^{1}$. By properties of the coproduct there is a continuous map $\sigma: s^{-1}(r(E)) \sqcup \bar{F} \rightarrow$ $r(E)$ with $\sigma(v)=v$ for $v \in \bar{F}$ and $\sigma(e)=s(e)$ for $e \in s^{-1}(r(E))$. Define $E^{1}$ as the pullback of the continuous maps $\sigma: s^{-1}(r(E)) \sqcup \bar{F} \rightarrow r(E)$ and $r: E \rightarrow r(E)$; i.e., $E^{1}=\left\{(e, f) \in E \times\left[s^{-1}(r(E)) \sqcup \bar{F}\right] \mid r(e)=\right.$ $\sigma(f)\}$ equipped with the continuous maps $s_{1}$ and $r_{1}$ from $E^{1}$ to $X^{1}$ which are the restrictions of the canonical projection maps on $E \times\left[s^{-1}(r(E)) \sqcup \bar{F}\right]$, so $s_{1}(e, f)=e$ and $r_{1}(e, f)=f$ for $(e, f) \in E^{1}$. Note that $r_{1}\left(E^{1}\right)=$ $s^{-1}(r(E)) \sqcup \bar{F}$. Finally, define a family $\lambda_{e}^{1}, e \in X^{1}=E \sqcup \bar{F}$ of Radon measures on $E^{1}$ by $\lambda_{e}^{1}=\lambda_{s(e)}$ for $e \in s^{-1}(r(E)), \lambda_{v}^{1}=\lambda_{v}$ for $v \in \bar{F}$ and zero otherwise. Since $E^{1} \subseteq X^{1} \times X^{1}$ it is a relation on the set $X^{1}$.

The case with $F$ the empty set corresponds to the situation that the restricted C*-correspondence $\mathscr{R}\left(\mathscr{E}_{G}\right)$ has an injective left action $\phi_{I}$. Then $\bar{F}$ is empty 
and $G^{1}=\left(X^{1}, E^{1}, r_{1}, s_{1}, \lambda^{1}\right)$ with $X^{1}=E$, and $E^{1}=\left\{(e, f) \in r^{-1}(s(E)) \times\right.$ $\left.s^{-1}(r(E)) \mid r(e)=s(f)\right\}$.

Proposition 2.2. The set $E^{1}$ is closed in $X^{1} \times X^{1}$.

Proof. If $\left(e_{n}, f_{n}\right) \rightarrow(e, f)$ with $e_{n} \in E, f_{n} \in\left[s^{-1}(r(E)) \sqcup \bar{F}\right]$ then, since $E$ is closed in $X^{1}$, we have $e \in E$, and by continuity $r\left(e_{n}\right) \rightarrow r(e)$. If $f \in E$, then $f_{n} \in E$ eventually, and by continuity $s\left(f_{n}\right) \rightarrow s(f)$. Since $s\left(f_{n}\right)=\sigma\left(f_{n}\right)=r\left(e_{n}\right)$ we must have $r(e)=s(f)$, so $f \in s^{-1}(r(E))$ and $(e, f) \in E^{1}$. If $f \in \bar{F}$ then $f_{n} \in \bar{F}$ eventually and $r\left(e_{n}\right)=\sigma\left(f_{n}\right)=f_{n} \rightarrow$ $f=\sigma(f)$, so $r(e)=\sigma(f)$ and $(e, f) \in E^{1}$.

In fact, since $E^{1}$ is contained in $E \times X^{1}$ we have that $E^{1}$ is also closed in $E \times X^{1}$. Note that $E^{1}$ is contained in the disjoint union $\left[E \times s^{-1}(r(E))\right] \cup$ $\left[r^{-1}(\bar{F}) \times \bar{F}\right]$.

For $X, Y$ locally compact Hausdorff spaces define a bilinear map $\Phi$ : $C_{c}(X) \times C_{c}(Y) \rightarrow C_{c}(X \times Y)$ by $\Phi(f, g)(x, y)=f(x) g(y),(x, y) \in X \times Y$. In the following Lemma the map $\varphi: C_{c}(X) \otimes C_{c}(Y) \rightarrow C_{c}(X \times Y)$ is the linear map with $\varphi(f \otimes g)=\Phi(f, g)$ and $\pi_{1}, \pi_{2}$ the canonical projection maps of $X \times Y$ to $X$ and $Y$ respectively.

Lemma 2.3. Let $X, Y$ be locally compact Hausdorff spaces, $f \in C_{c}(X \times Y)$ with $\operatorname{supp}(f)=K$ and $\epsilon>0$. There is an element $g$ in the (algebraic) tensor product $C_{\pi_{1}(K)}(X) \otimes_{\text {alg }} C_{\pi_{2}(K)}(Y)$ with $\operatorname{supp}(\varphi(g)) \subseteq \operatorname{supp}(f)$ and $\|f-\varphi(g)\|_{\infty}<\epsilon$.

Proof. It is enough to show this for positive functions $f$. Let $U_{l} \times V_{k}$ be a finite covering of $\pi_{1}(K) \times \pi_{2}(K)$ by open rectangles with compact closures on which the function $f$ varies by less than $\epsilon / 2$. Then use a partition of unity $\alpha_{l}, \beta_{k}$ subordinate to $U_{l}$ and $V_{k}$ respectively in each of $C_{\pi_{1}(K)}(X)$ and $C_{\pi_{2}(K)}(Y)$, and set $g=\sum \delta_{l . k} \alpha_{l} \otimes \beta_{k}$ where $\delta_{l, k}=\inf \left\{f(x, y) \mid(x, y) \in U_{l} \times V_{k}\right\}$, so that the set of points where $\varphi(g)$ is nonzero is contained in the set of points where $f$ is nonzero. We have that $f(x, y)$ is within $\epsilon$ of $\delta_{l . k}$ on $U_{l} \times V_{k}$ and since $\varphi\left(\sum \alpha_{l} \otimes \beta_{k}\right)=1$ on $K$ the result follows.

Proposition 2.4. For $\xi \in C_{c}\left(E^{1}\right)$ there are compact subsets $K_{1}, K_{2}$ of $E$ and $X^{1}$ respectively such that for any $\epsilon>0$ there is $\xi_{0} \in C_{c}\left(E^{1}\right)$ with $\left\|\xi-\xi_{0}\right\|_{\infty}<\epsilon, \operatorname{supp}\left(\xi_{0}\right) \subseteq \operatorname{supp}(\xi)$, and $\xi_{0}=i^{\#} \varphi(g)$ the restriction to $E^{1}$ of an element $\varphi(g)$ in $C_{c}\left(E \times X^{1}\right)$ with $g \in C_{K_{1}}(E) \otimes_{\mathrm{alg}} C_{K_{2}}\left(\left[s^{-1}(r(E)) \sqcup \bar{F}\right]\right)$.

Proof. Since $E^{1}$ is closed in $E \times X^{1}$ the Tietze Extension Theorem and Urysohn's Lemma yield an element in $f \in C_{c}\left(E \times X^{1}\right)$ whose restriction to $E^{1}$ is $\xi$. Since $r$ is an open map, $E \times\left[s^{-1}(r(E)) \sqcup \bar{F}\right]$ is open in $E \times X^{1}$ and there is an open set $V$ containing $\operatorname{supp}(\xi)$ with $\bar{V}$ compact and contained in 
$E \times\left[s^{-1}(r(E)) \sqcup \bar{F}\right]$. We can choose $V$ so that $\bar{V}$ is contained in any given neighbourhood of $\operatorname{supp}(\xi)$. The function $f$ can be chosen with $\operatorname{supp}(f) \subseteq \bar{V}$. Let $K_{i}=\pi_{i}(\bar{V})$. Apply the previous lemma to obtain $g$ and set $\xi_{0}$ to be the restriction of $\varphi(g)$ to $E^{1}$ as required. The proof of this lemma also shows that $\left\{a \in E^{1} \mid \xi_{0}(a) \neq 0\right\}$ is contained in the set of elements of $E^{1}$ where $f$, so $\xi$, is nonzero, and the support condition follows.

THEOREM 2.5. If $G=(X, E, r, s, \lambda)$ is a topological quiver then the associated relation $G^{1}=\left(X^{1}, E^{1}, r_{1}, s_{1}, \lambda^{1}\right)$ is a topological quiver.

Proof. First we show the map $r_{1}$ is open since it is defined using the pullback of the open map $r$; if $O \subseteq E^{1}$ is open and $(a, b) \in O$ we show that there is an open set $V$ in $s^{-1}(r(E)) \sqcup \bar{F}$ with $b=r_{1}(a, b) \in V \subseteq r_{1}(O)$. First note there are neighbourhoods $O_{a}, O_{b}$ of $a, b$ respectively with $E^{1} \cap O_{a} \times O_{b} \subseteq$ $O$. Since $r$ is open $r\left(O_{a}\right)$ is open and $b$ is in the open set $V=O_{b} \cap \sigma^{-1}\left(r\left(O_{a}\right)\right)$. To see $V \subseteq r_{1}(O)$ choose $f \in V$; then $f \in O_{b}$ and there is an $e \in O_{a}$ with $\sigma(f)=r(e)$. Thus $(e, f) \in E^{1} \cap O_{a} \times O_{b}$ and $f \in r_{1}\left(E^{1} \cap O_{a} \times O_{b}\right) \subseteq r_{1}(O)$. Thus $V \subseteq r_{1}(O)$ and $b=r_{1}(a, b) \in V \subseteq r_{1}(O)$.

For $b \in s^{-1}(r(E)) \sqcup \bar{F},\left(r_{1}\right)^{-1}(b)=\left\{(a, b) \in E^{1} \mid a \in E, r(a)=\right.$ $\sigma(b)\}=r^{-1}(\sigma(b)) \times\{b\}$, while $\left(r_{1}\right)^{-1}(b)=\phi$ otherwise. Thus $\operatorname{supp}\left(\lambda_{b}^{1}\right)=$ $\operatorname{supp}\left(\lambda_{\sigma(b)}\right)=r^{-1}(\sigma(b))=\left(r_{1}\right)^{-1}(b)$.

For $\xi \in C_{c}\left(E^{1}\right)$ we need to show that the map on $X^{1}$ sending $b$ to $H(\xi)(b)=$ $\int_{E^{1}} \xi(e, f) d \lambda_{b}^{1}((e, f))$ is continuous. We have $H(\xi)(b)=\int_{r_{1}^{-1}(b)} \xi(e, f)$ $d \lambda_{b}^{1}((e, f))=\int_{r^{-1}(\sigma(b))} \xi(e, b) d \lambda_{\sigma(b)}(e)$. Choose $K_{1}, K_{2}$ compact sets of $E$ and $X^{1}$ respectively, and for $\epsilon>0$, choose $\xi_{0}=\varphi(k)$ with $k$ in $C_{K_{1}}(E) \otimes$ $C_{K_{2}}\left(X^{1}\right)$ as in the preceding proposition. If $\xi^{b}$ denotes the function in $C_{K_{1}}(E)$ defined by $\xi^{b}(e)=\xi(e, b)$ we have by Remark $2.1\left\|H(\xi)(b)-H\left(\xi_{0}\right)(b)\right\|=$ $\left\|\lambda_{\sigma(b)}\left(\xi^{b}-\xi_{0}^{b}\right)\right\| \leq N_{K_{1}}\left\|\xi^{b}-\xi_{0}^{b}\right\|_{\infty} \leq N_{K_{1}}\left\|\xi-\xi_{0}\right\|_{\infty}$, which is independent of $b$. However, for $\xi_{0}=\varphi(k)$ with $k$ a simple tensor $g \otimes h \in C_{K_{1}}(E) \otimes C_{K_{2}}\left(X^{1}\right)$ compute that $H\left(\xi_{0}\right)(b)=h(b) \int g(e) d \lambda_{\sigma(b)}(e)$; this is the product of an element in $C_{K_{1}}(E)$ and an element in $\sigma^{\#}\left(C_{c}(X)\right) \subseteq C_{b}\left(X^{1}\right)$, so is in $C_{K_{1}}(E)$. Thus $H(\xi) \in C_{K_{1}}(E)$.

Lemma 2.6. If $h: E \rightarrow X$ is an open map of topological spaces and $A \subseteq X$, then $h^{-1}(A) \cup h^{-1}(\bar{A})^{c}$ is dense in $E$.

Proof. If not, there is an open set $N \subseteq E$ with $N \subseteq E-\left[h^{-1}(A) \cup\right.$ $\left.h^{-1}(\bar{A})^{c}\right]=h^{-1}\left(A^{c}\right) \cap h^{-1}(\bar{A})$. Thus the open set $h(N) \subseteq A^{c} \cap \bar{A}$, so $h(N) \subseteq$ $A^{c}$ and $h(N) \cap A \neq \phi$, a contradiction.

Proposition 2.7. If $G=(X, E, r, s, \lambda)$ is a topological quiver with associated topological relation $G^{1}=\left(X^{1}, E^{1}, r_{1}, s_{1}, \lambda^{1}\right)$ then $\bar{F}=X_{\text {sink }}^{1}$, the set of sinks of $G^{1}$. 
Proof. The set $X_{\text {sink }}^{1}=X^{1}-\overline{s_{1}\left(E^{1}\right)}=(E \sqcup \bar{F})-\overline{s_{1}\left(E^{1}\right)}$, and since $E^{1} \subseteq E \times\left[s^{-1}(\underline{r(E))} \sqcup \bar{F}], s_{1}\left(E^{1}\right)=\left\{e \mid(e, f) \in E^{1}\right\} \subseteq E\right.$. Now $E$ is closed in $X^{1}$, so $s_{1}\left(E^{1}\right) \subseteq E$ and $X_{\text {sink }}^{1}=\left[E-\overline{s_{1}\left(E^{1}\right)}\right] \sqcup \bar{F}$.

The image $\sigma\left[s^{-1}(r(E)) \sqcup \bar{F}\right]=s\left(s^{-1}(r(E))\right) \sqcup \bar{F}=[s(E) \cap r(E)] \sqcup \bar{F}$, so $s_{1}\left(E^{1}\right)=r^{-1}(\operatorname{Im}(\sigma))=r^{-1}([s(E) \cap r(E)] \sqcup \bar{F})=r^{-1}([s(E) \cap r(E)]) \cup$ $\left.r^{-1}(\bar{F}) \supseteq r^{-1}(s(E))\right) \cup r^{-1}\left(\overline{s(E)}^{c}\right)$. The preceding lemma with $h=r$ and $A=s(E)$ shows $s_{1}\left(E^{1}\right)$ is dense in $E$, so $X_{\text {sink }}^{1}=\bar{F}$.

Let $\mathscr{E}^{1}=\mathscr{E}_{G^{1}}$ denote the $\mathrm{C}^{*}$-correspondence associated with the topological relation $G^{1}$. Then the ideal $I_{1}=\overline{\langle\mathscr{E} 1, \mathscr{E} 1\rangle}=C_{0}\left(r_{1}\left(E^{1}\right)\right)$ where the image of $r_{1}$ is $\left\{f \mid(e, f) \in E^{1}\right\}=\left\{f \mid f \in s^{-1}(r(E)) \sqcup \bar{F}, \sigma(f) \in r(E)\right\}=$ $s^{-1}(r(E)) \sqcup \bar{F}_{G}$, so the restricted correspondence $\mathscr{R}(\mathscr{E} 1)=I_{I_{1}}\left(\mathscr{E}^{1}\right)_{I_{1}}$ is a bimodule over the $\mathrm{C}^{*}$-algebra $C_{0}\left(s^{-1}(r(E)) \sqcup \bar{F}\right)=C_{0}\left(s^{-1}(r(E))\right) \oplus C_{0}(\bar{F})$. Since the kernel, $\operatorname{ker}\left(\left(\phi_{1}\right)_{I_{1}}\right)$ of the restricted left multiplication map for the correspondence $\mathscr{R}\left(\mathscr{E}^{1}\right)$ is $C_{0}\left(F^{1}\right)$ where $F^{1}\left(=F_{G^{1}}\right)=r_{1}\left(E^{1}\right) \cap X_{\text {sink }}^{1}$ the proposition implies $F^{1}=\left(s^{-1}(r(E)) \sqcup \bar{F}\right) \cap \bar{F}=\bar{F}$, so $\operatorname{ker}\left(\left(\phi_{1}\right)_{I_{1}}\right)=C_{0}(\bar{F})$.

Corollary 2.8. If $G=(X, E, r, s, \lambda)$ is a topological quiver with associated topological relation $G^{1}=\left(X^{1}, E^{1}, r_{1}, s_{1}, \lambda^{1}\right)$ then the associated restricted correspondence $\mathscr{R}(\mathscr{E})$ has an injective left action $\phi_{I}$ if and only if the left action $\left(\phi_{1}\right)_{I_{1}}$ for the correspondence $\mathscr{R}\left(\mathscr{E}^{1}\right)$ is injective.

The map $\sigma^{\#}: C_{0}(r(E)) \rightarrow C_{b}\left(s^{-1}(r(E)) \sqcup \bar{F}\right)$ defines a left action of the $\mathrm{C}^{*}$-algebra $I=C_{0}(r(E))$ on the $\mathrm{C}^{*}$-algebra $I_{1}=C_{0}\left(s^{-1}(r(E)) \sqcup \bar{F}\right)=$ $C_{0}\left(s^{-1}(r(E)) \oplus C_{0}(\bar{F})\right.$, allowing $I_{1}$ to be viewed as a $C^{*}$-correspondence from $I$ to $I_{1}$.

There is also a left action of $I_{1}$ on the Hilbert module $\mathscr{E}_{I}$. Note first that $C_{0}(E)$ acts by left multiplication on the (nonclosed) ideal $C_{c}(E)$ and since $C_{0}\left(s^{-1}(r(E))\right.$ is an ideal of $C_{0}(E)$ there is a left multiplication action of $C_{0}\left(s^{-1}(r(E))\right.$ on $C_{c}(E)$. Since the $I$-Hilbert module structure on $\mathscr{E}_{G}$ is defined using the integral of a product of functions in $C_{c}(E)$, the left multiplication action of $C_{0}\left(s^{-1}(r(E))\right.$ on the Hilbert module $\mathscr{E}$ is by adjointable operators. Letting $C_{0}(\bar{F})$ act as 0 on $\mathscr{E}$ yields a $C^{*}$-correspondence ${ }_{I_{1}} \mathscr{E}_{I}$ from $I_{1}=$ $C_{0}\left(s^{-1}(r(E)) \oplus C_{0}(\bar{F})\right.$ to $I$.

THEOREM 2.9. Let $G=(X, E, r, s, \lambda)$ be a topological quiver with associated correspondence $\mathscr{E}_{G}=\mathscr{E}$ and $G^{1}=\left(X^{1}, E^{1}, r_{1}, s_{1}, \lambda^{1}\right)$ the associated topological relation with correspondence $\mathscr{E}_{G^{1}}=\mathscr{E}^{1}$. Then the restricted correspondence $\mathscr{R}(\mathscr{E} 1)=I_{I_{1}}(\mathscr{E} 1)_{I_{1}}$ is isomorphic to the interior tensor product $I_{1} \mathscr{E}_{I} \otimes_{I}\left(I_{1}\right)_{I_{1}}$ of $C^{*}$-correspondences.

Proof. With $i: E^{1} \rightarrow E \times r_{1}\left(E^{1}\right)$ the inclusion and $\varphi$ the linear map defined on the algebraic tensor product in Lemma 2.3 form the linear map 
$i^{\#} \circ \varphi: C_{c}(E) \otimes_{\mathrm{alg}} C_{c}\left(r_{1}\left(E^{1}\right)\right) \rightarrow C_{c}\left(E^{1}\right)$. For $g \otimes h$ a simple tensor in the algebraic tensor product, $a \in I$ and $(e, f) \in E^{1}$ we have $i^{\#} \varphi(g a \otimes h-$ $\left.g \otimes \sigma^{\#}(a) h\right)(e, f)=(g a)(e) h(f)-g(e)\left(\sigma^{\#}(a) h\right)(f)=g(e) a(r(e)) h(f)-$ $g(e) a(\sigma(f)) h(f)=0$ since $r(e)=\sigma(f)$ for $(e, f) \in E^{1}$. This defines a well defined linear map $\gamma$ from the quotient of the algebraic tensor product by the subspace $N$ generated by $\left\{g a \otimes h-g \otimes a h \mid a \in I, g \in C_{c}(E), h \in\right.$ $\left.C_{c}\left(r_{1}\left(E^{1}\right)\right)\right\}$, so from the pre-Hilbert $I_{1}$-module interior algebraic tensor product $C_{c}(E)_{I} \otimes_{\text {alg } I} C_{c}\left(r_{1}\left(E^{1}\right)\right)$, to $C_{c}\left(E^{1}\right)$.

Using the description of the map $H$ on simple tensors in the proof of Theorem 2.5 and computing we have $\langle\gamma(g \otimes h), \gamma(k \otimes l)\rangle=H\left(i^{\#} \varphi(\bar{g} \otimes \bar{h}) i^{\#} \varphi(k \otimes\right.$ $l))=H\left(i^{\#} \varphi(\bar{g} k \otimes \bar{h} l)\right)=\bar{h} l\langle g, k\rangle_{I}$ which is, by definition of the interior tensor inner product, equal to $\langle g \otimes h, k \otimes l\rangle_{I_{1}}$ ([11]). Thus $\gamma$ defines an isometry, denoted $\gamma$ still, of Hilbert $I_{1}$-modules. The map $i^{\#} \circ \varphi$ has dense range in $C_{c}\left(E^{1}\right)$ by Remark 2.1 and Proposition 2.4, so $\gamma$ is a surjective isometry.

For $b=\left(b_{1}, b_{2}\right) \in I_{1}=C_{0}\left(s^{-1}(r(E)) \oplus C_{0}(\bar{F}), g \otimes h\right.$ a simple tensor in $C_{c}(E)_{I} \otimes_{\text {alg } I} C_{c}\left(r_{1}\left(E^{1}\right)\right)$ and $(e, f) \in E^{1}$ we have $(\gamma(g \otimes h) b)(e, f)=$ $\gamma(g \otimes h)(e, f) b\left(r_{1}(e, f)\right)=g(e) h(f) b(f)=\gamma(g \otimes(h b))(e, f)=\gamma((g \otimes$ $h) b)(e, f)$. We also have $[\gamma(b(g \otimes h))](e, f)=\gamma((b \cdot g) \otimes h))(e, f)=$ $\left(b_{1} g\right)(e) h(f)=b_{1}(e) g(e) h(f)$ which, since $s_{1}\left(E^{1}\right) \subseteq E$, is equal to $\left(b s_{1}(e\right.$, $f)) \gamma(g \otimes h)(e, f)=[b \cdot \gamma(g \otimes h)](e, f)$. It follows that both $\gamma(x b)=\gamma(x) b$ and $\gamma(b x)=b \gamma(x)$ for $x \in \mathscr{E}_{I} \otimes_{I}\left(I_{1}\right)$ and $b \in I_{1}$, so that $\left(\gamma, \operatorname{Id}_{I_{1}}\right)$ is an isomorphism of the stated correspondences.

We use the isomorphism of correspondences in Theorem 2.9 to recast how we view the left action $\left(\phi_{1}\right)_{I_{1}}(a)$ on $\mathscr{R}\left(\mathscr{E}^{1}\right)$ for $a \in I_{1}$. Denote the left multiplication action of $C_{0}\left(s^{-1}(r(E))\right.$ on the Hilbert module $\mathscr{E}_{I}$, the completion of $C_{c}(E)$, by $M_{a}$ for $a \in C_{0}\left(s^{-1}(r(E))\right.$. In fact this multiplication action is the restriction to the ideal $C_{0}\left(s^{-1}(r(E))\right.$ of the multiplication action $M$ of $C_{b}(E)$ by adjointable operators on (the completion of) $C_{c}(E)$ ([14] Lemma 3.6). If $j$ is the inclusion $s^{-1}(r(E))$ into $s^{-1}(r(E)) \sqcup \bar{F}$ then the left action of $I_{1}$ on the Hilbert module $\mathscr{E}_{I}$ is the left multiplication action $M_{j^{\sharp} a}$ on $\mathscr{E}$, for $a \in C_{0}\left(s^{-1}(r(E)) \oplus C_{0}(\bar{F})\right.$. Therefore we may view $\left(\phi_{1}\right)_{I_{1}}(a)$ on $\mathscr{R}(\mathscr{E} 1)$ as equal to $M_{j^{\sharp} a} \otimes \operatorname{Id}_{I_{1}}$ on $I_{I_{1}}^{\mathscr{E}} \mathscr{E}_{I} \otimes_{I}\left(I_{1}\right)_{I_{1}},\left(a \in I_{1}\right)$.

REMARK 2.10. This tensor product characterization of the correspondence of the multiplicity free quiver $\mathscr{R}\left(\mathscr{E}^{1}\right)$ provides a means to define $\mathscr{R}\left(\mathscr{E}^{1}\right)$ for a general restricted $\mathrm{C}^{*}$-correspondence $\mathscr{R}(\mathscr{E})$ not necessarily arising from a topological quiver. 


\section{Proper quivers}

We introduce the concept of a proper topological quiver $G$, and show that if $G$ is proper then there is an injection of the restricted $\mathrm{C}^{*}$-correspondence $\mathscr{R}(\mathscr{E})$ to $\mathscr{R}\left(\mathscr{E}^{1}\right)$.

For $A, B$ given $\mathrm{C}^{*}$-algebras and $\varphi: A \rightarrow M(B)$ a $*$-homomorphism we are interested in isolating what we term the proper part $\varphi_{p}$ of $\varphi$, namely the aspect of $\varphi$ mapping into $B$.

If $\pi: M(B) \rightarrow M(B) / B$ is the canonical quotient map onto the corona algebra with kernel $B$ we set $A_{p}$ to be kernel of $\pi \circ \varphi$, an ideal of $A$. It is clear that $\varphi$ restricted to $A_{p}$ maps into $\operatorname{ker} \pi=B$. Denote by $B_{p}$ the smallest ideal of $B$ containing the image $C^{*}$-algebra $\varphi\left(A_{p}\right)$ and $\varphi_{p}: A_{p} \rightarrow B_{p}$ the restriction of $\varphi$ to domain $A_{p}$ and codomain $B_{p}$. Note that if $B$ is unital then $A_{p}=A$.

Definition 3.1. For a continuous map $s: E \rightarrow X$ of locally compact Hausdorff spaces let $X_{p}(s)=\left\{x \in X \mid \exists\right.$ a neighbourhood $N_{x}$ of $x$ with both $\overline{N_{x}}$ and $s^{-1}\left(\overline{N_{x}}\right)$ compact\}. If the context is clear $X_{p}$ is used.

The set $X_{p}$ is open in $X$ and always contains the open set $X-\overline{s(E)}$ so a set $U$ is in $X_{p}$ if and only if $U \cap \overline{s(E)} \subseteq X_{p}$.

If $K \subseteq X_{p}$ is compact then $s^{-1}(K)$ is compact in $E$, since it is a closed subset of the compact set $\bigcup_{i \in B} s^{-1}\left(\overline{N_{x_{i}}}\right)$ where $\left\{N_{x_{i}} \mid i \in B\right\}$ is a finite subcover of the open cover $\left\{N_{x} \mid x \in K\right\}$ of $K, N_{x}$ chosen with $s^{-1}\left(\overline{N_{x}}\right)$ compact. If $U$ is an open subset of $X_{p}$ and $K \subseteq U$ the set $s^{-1}(K) \subseteq s^{-1}(U)$ so is also compact in the subspace $s^{-1}(U)$. Therefore the map of locally compact Hausdorff spaces given by the restriction of $s$ to domain $s^{-1}(U)$ and codomain $U$ is a proper map. Thus $X_{p}(s)$ is the largest open set $U$ of $X$ so that $s: s^{-1}(U) \rightarrow U$ is a proper map. Note that if $E$ is a compact space then $s$ is a proper map and $X_{p}=X$.

Lemma 3.2. Let $s: E \rightarrow X$ be a continuous map of locally compact Hausdorff spaces and $Y \subseteq X_{p}(s)$. Then $Y \cap s(E)=Y \cap \overline{s(E)}$.

Proof. For $x \in Y \cap \overline{s(E)}$, choose a neighbourhood $N_{x}$ of $x$ with both $\overline{N_{x}}$ and $s^{-1}\left(\overline{N_{x}}\right)$ compact. If $e_{n}$ is a net with $s\left(e_{n}\right) \rightarrow x$ then there is an $n_{0}$ with $e_{n} \in s^{-1}\left(N_{x}\right)$, so in the compact set $s^{-1}\left(\overline{N_{x}}\right)$, for $n_{0} \prec n$. There is a convergent subnet $e_{n_{i}} \rightarrow e \in s^{-1}\left(\overline{N_{x}}\right)$, so $s\left(e_{n_{i}}\right) \rightarrow s(e)$. Thus $x=s(e) \in r(E) \cap s(E)_{p}$.

Theorem 3.3. If $s: E \rightarrow X$ is continuous map of locally compact Hausdorff spaces and $\varphi: C_{0}(X) \rightarrow C_{b}(E)$ is the induced map $s^{\#}$, then $\varphi_{p}: C_{0}\left(X_{p}\right) \rightarrow C_{0}\left(s^{-1}\left(X_{p}\right)\right)$ is the proper part of $\varphi$ with $\varphi_{p}=\left(s_{p}\right)^{\#}, s_{p}$ the proper map given by restricting $s$ to domain $s^{-1}\left(X_{p}\right)$ and codomain $X_{p}$.

Proof. Let $\pi$ denote the projection of $C_{b}(E)=M\left(C_{0}(E)\right)$ to the corona algebra $C_{b}(E) / C_{0}(E)$. We first show that $C_{0}\left(X_{p}\right)$ is the ideal $C_{0}(X)_{p}=$ 
$\operatorname{ker}(\pi \circ \varphi)$. To show the containment $C_{0}\left(X_{p}\right) \subseteq \operatorname{ker}(\pi \circ \varphi)$ it is enough to show that $\varphi(f) \in C_{c}(E)$ for $f \in C_{c}\left(X_{p}\right)$. However if $\operatorname{supp}(f)=K$ is compact in $X_{p}$ then by the above comment $s^{-1}(K)$ is compact in $E$ and clearly $\varphi(f)=f \circ s$ has support in $s^{-1}(K)$. Thus if $O$ is the open set in $X$ with $C_{0}(O)=C_{0}(X)_{p}$ we have that $X_{p} \subseteq O$. Suppose that there is an $x \in O-X_{p}$. Then there are neighbourhoods $N, M$ of $x$ with compact closures and $\bar{N} \subset M \subset \bar{M} \subset O$. By Urysohn's Lemma there is an $f \in C_{c}(O) \subseteq C_{0}(X)_{p}$ with $0 \leq f \leq 1, f$ is 1 on $\bar{N}$ and $\operatorname{supp}(f) \subseteq \bar{M}$. Since $x \notin X_{p}, s^{-1}(\bar{N})$ is not compact in $E$ and $\varphi(f)$, which is identically 1 on this noncompact set cannot be in $C_{0}(E)=\operatorname{ker}(\pi)$. This contradicts $f \in \operatorname{ker}(\pi \circ \varphi)$, so $O=X_{p}$ and $C_{0}\left(X_{p}\right)=C_{0}(X)_{p}$.

The map $s_{p}$ is a proper map and $\left(s_{p}\right)^{\#}: C_{0}\left(X_{p}\right) \rightarrow C_{0}\left(s^{-1}\left(X_{p}\right)\right)$. Therefore $C_{0}(E)_{p} \subseteq C_{0}\left(s^{-1}\left(X_{p}\right)\right)$. Writing $C_{0}(E)_{p}$ as $C_{0}(U)$ for some open set $U$ in $E$ we have $U \subseteq s^{-1}\left(X_{p}\right)$. If this inclusion is proper then there is an $e \in s^{-1}\left(X_{p}\right)-$ $U$. Chose a neighbourhood $N$ of $x=s(e)$ with $\bar{N}$ and $s^{-1}(\bar{N})$ compact and $\bar{N} \subseteq X_{p}$. Applying Urysohn's Lemma again yields a $f \in C_{c}\left(X_{p}\right), 0 \leq f \leq 1$, $f(x)=1$, and $\operatorname{supp}(f) \subseteq \bar{N}$. Since $\varphi(f)(e)=1$, it follows that $\varphi(f) \notin$ $C_{0}(U)$, contrary to how $C_{0}(E)_{p}$ is defined, so $C_{0}(E)_{p}=C_{0}\left(s^{-1}\left(X_{p}\right)\right)$.

Definition 3.4. A topological quiver $G=(X, E, r, s, \lambda)$ is proper if $r(E) \subseteq X_{p}(s)$. Denote by $s_{p}$ the restriction of $s$ to domain $s^{-1}(r(E))$ and codomain $r(E)$.

Note that the open set $X_{\text {fin }} \subseteq X_{p}(s)$. Since $r$ is an open map, the comment following Definition 3.1 implies that a quiver $G=(X, E, r, s, \lambda)$ is proper if $r(E) \cap \overline{s(E)} \subseteq X_{p}$. If $G$ is a discrete directed graph then $X_{\text {fin }}=X_{p}(s)$, and $G$ is proper if all the vertices which are not sources emit a finite number of edges. If the quiver arises from a continuous map $f: X \rightarrow Y$ then it is proper if and only if the map $f$ is a proper map in the usual sense.

For $G$ proper, the map $s_{p}: s^{-1}(r(E)) \rightarrow r(E)$ is a proper map by the last theorem. Also, from Lemma 3.2 we have that $r(E) \cap \overline{s(E)}=r(E) \cap s(E)$ and so $s(E)$ is closed in the subspace $r(E)$.

Lemma 3.5. If $G=(X, E, r, s, \lambda)$ is a proper topological quiver then the restriction of $\sigma, \sigma_{p}: s^{-1}(r(E)) \sqcup \bar{F} \rightarrow r(E)$ is a proper surjection.

Proof. Let $K$ be a compact subset of $r(E)$. Recalling that $\bar{F}$ denotes the closure of $F=r(E)-\overline{s(E)}$ in $r(E)$, we have that $K \cap \bar{F}$ is a closed subset of the compact set $K$, so is compact in $r(E)$. Since $K \cap \bar{F}$ is contained in $\bar{F}$ it is also compact in the subspace $\bar{F}$. We have $\left(\sigma_{p}\right)^{-1}(K)=\left(s_{p}\right)^{-1}(K) \sqcup(K \cap \bar{F})$ which is compact in $s^{-1}(r(E)) \sqcup \bar{F}$. We have $r(E)=(r(E) \cap \overline{s(E)}) \cup F=$ $(r(E) \cap \overline{s(E)}) \cup \bar{F}$. By Lemma 3.2 this is $(r(E) \cap s(E)) \cup \bar{F}$ and since $s_{p}$ is onto $r(E) \cap s(E)$ and the identity maps onto $\bar{F}$ we see $\sigma_{p}$ is onto. 
In particular note that if $r(E)$ is compact then this implies that $s^{-1}(r(E)) \sqcup \bar{F}$, and so $s^{-1}(r(E))$ must be compact.

Proposition 3.6. Let $G=(X, E, r, s, \lambda)$ be a proper topological quiver and $G^{1}=\left(X^{1}, E^{1}, r_{1}, s_{1}, \lambda^{1}\right)$ the associated topological relation. With $\mathscr{E}=$ $\mathscr{E}_{G}$ and $\mathscr{E}^{1}=\mathscr{E}_{G^{1}}$ the pair $\left(s_{1}^{\#}, \sigma_{p}^{\#}\right): \mathscr{R}(\mathscr{E}) \rightarrow \mathscr{R}(\mathscr{E} 1)$ defines an injective morphism of $C^{*}$-correspondences.

Proof. It follows from the above lemma that $\sigma_{p}^{\#}: C_{0}(r(E)) \rightarrow$ $C_{0}\left(s^{-1}(r(E)) \oplus C_{0}(\bar{F})\right.$ is an injective ${ }^{*}$-homomorphism of $\mathrm{C}^{*}$-algebras, $\sigma_{p}^{\#}$ : $I \rightarrow I_{1}$.

General properties of the pullback construction of $E^{1}$ imply $s_{1}: E^{1} \rightarrow E$ is also proper, for if $K$ is a compact subset of $E$ then $r(K)$ is compact and since $\sigma_{p}$ is proper, $\sigma_{p}^{-1}(r(K))$ is compact in $E \sqcup \bar{F}$. Since the set $\left(s_{1}\right)^{-1}(K)$ is a closed subset of the compact set $K \times \sigma_{p}^{-1}(r(K))$ it is compact. Also $s_{1}$ is surjective since $\sigma_{p}$ is. Thus $\left(s_{1}\right)^{\#}: C_{c}(E) \rightarrow C_{c}\left(E^{1}\right)$ is an injective *-homomorphism.

We need to verify the first two conditions that a morphism of correspondences must satisfy (Definition 1.1). For $g, h \in C_{c}(E)$ and for $f \in \sigma_{p}^{-1}(r(E))$ we have that

$$
\begin{aligned}
\left.\left\langle\left(s_{1}\right)^{\#}(g),\left(s_{1}\right)^{\#}(h)\right)\right\rangle_{I_{1}}(f) & =\int_{\left(r_{1}\right)^{-1}(f)} \overline{\left(s_{1}\right)^{\#}(g)}(e, f)\left(s_{1}\right)^{\#}(h)(e, f) d \lambda_{f}^{1}(e, f) \\
& =\int_{r^{-1}\left(\sigma_{p}(f)\right)} \overline{g(e)} h(e) d \lambda_{\sigma_{p}(f)}(e) \\
& =\langle g, h\rangle_{I}\left(\sigma_{p}(f)\right)=\sigma_{p}^{\#}\langle g, h\rangle_{I}(f),
\end{aligned}
$$

so $\left(s_{1}\right)^{\#}$ is an isometry of pre-Hilbert modules and extends to a well defined isometry of their completions. To see $\left(s_{1}\right)^{\#}\left(\phi_{I}(a) g\right)=\left[\left(\phi_{1}\right)_{I_{1}}\left(\sigma_{p}^{\#} a\right)\right]\left[\left(s_{1}\right)^{\#}(g)\right]$ evaluate at $(e, f) \in E^{1}$. The left side is equal to $\left(\phi_{I}(a) g\right)(e)=a\left(\sigma_{p}(e)\right) g(e)$ while the right side is $\left(\sigma_{p}^{\#}(a)\right)(e)\left(g\left(s_{1}(e, f)\right)\right)=a\left(\sigma_{p}(e)\right) g(e)$.

We consider the ${ }^{*}$-homomorphism $\Psi_{\left(s_{1}\right)^{*}}: \mathscr{K}(\mathscr{E}) \rightarrow \mathscr{K}(\mathscr{E} 1)($ Remark 1.7) defined by the morphism $\left(s_{1}^{\#}, \sigma_{p}^{\#}\right): \mathscr{R}(\mathscr{E}) \rightarrow \mathscr{R}(\mathscr{E} 1)$ of correspondences.

Proposition 3.7. Let $G=(X, E, r, s, \lambda)$ be a proper topological quiver and $G^{1}=\left(X^{1}, E^{1}, r_{1}, s_{1}, \lambda^{1}\right)$ the associated topological relation. Under the

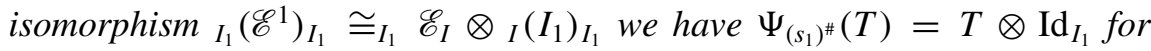
$T \in \mathscr{K}(\mathscr{E})$.

Proof. As in the last proposition $\sigma_{p}^{\#}: C_{0}(r(E)) \rightarrow C_{0}\left(s^{-1}(r(E))\right) \oplus$ $C_{0}(\bar{F})$ is an injective *-homomorphism of $\mathrm{C}^{*}$-algebras. Thus the left action of $I$ on $I_{1}$, which is given by the injection $\sigma_{p}^{\#}$, is by elements of $I_{1}=\mathscr{K}\left(I_{1}\right)$, so 
([11], Proposition 4.7) the map $T \rightarrow T \otimes \operatorname{Id}_{I_{1}}$ is an injective *-homomorphism of $\mathscr{K}(\mathscr{E}) \rightarrow \mathscr{K}(\mathscr{E} 1)$. To show this map equals $\Psi_{\left(s_{1}\right)^{\sharp}}$ it is enough to show both agree on the elements $\theta_{k, l} \in \mathscr{K}(\mathscr{E}), k, l \in C_{c}(E)$.

Note first that for $g \in C_{c}(E), h \in C_{c}\left(s^{-1}(r(E)) \oplus C_{c}(\bar{F})\right.$ and $(e, f) \in$ $E^{1}$ we have $(g \otimes h)(e, f)=g(e) h(f)=\left(s_{1}\right)^{\#}(g)(e, f)\left(r_{1}\right)^{\#}(h)(e, f)=$ $\left[\left(s_{1}\right)^{\#}(g) \cdot h\right](e, f)$, so $g \otimes h=\left(s_{1}\right)^{\#}(g) \cdot h$. Using this twice we compute $\left[\Psi_{\left(s_{1}\right)^{\#}}\left(\theta_{k, l}\right)\right](g \otimes h)=\left(\theta_{\left(s_{1}\right)^{\#}(k),\left(s_{1}\right)^{\#}(l)}\right)(g \otimes h)=\left(s_{1}\right)^{\#}(k) \cdot\left\langle\left(s_{1}\right)^{\#}(l), g \otimes\right.$ $h\rangle_{I_{1}}=\left(s_{1}\right)^{\#}(k) \cdot\left\langle\left(s_{1}\right)^{\#}(l),\left(s_{1}\right)^{\#} g \cdot h\right\rangle_{I_{1}}=\left(s_{1}\right)^{\#}(k) \cdot\left\langle\left(s_{1}\right)^{\#}(l),\left(s_{1}\right)^{\#} g\right\rangle_{I_{1}} h$. Since $\left(s_{1}\right)^{\#}$ is a Hilbert module morphism, the later is $\left(s_{1}\right)^{\#}(k) \cdot\left[\left(\sigma_{p}^{\#}\right)\left(\langle l, g\rangle_{I}\right)\right] h=$ $k \otimes\left[\left(\sigma_{p}^{\#}\right)\langle l, g\rangle_{I}\right] h=k \cdot\langle l, g\rangle_{I} \otimes h=\theta_{k, l}(g) \otimes h=\left(\theta_{k, l} \otimes \operatorname{Id}_{I_{1}}\right)(g \otimes h)$.

THeOREM 3.8. Let $G=(X, E, r, s, \lambda)$ be a proper topological quiver and $G^{1}=\left(X^{1}, E^{1}, r_{1}, s_{1}, \lambda^{1}\right)$ the associated topological relation. With $\mathscr{E}=\mathscr{E}_{G}$ and $\mathscr{E} 1=\mathscr{E}_{G^{1}}$ the morphism $\left(s_{1}^{\#}, \sigma_{p}^{\#}\right): \mathscr{R}(\mathscr{E}) \rightarrow \mathscr{R}(\mathscr{E} 1)$ of $C^{*}$-correspondences is coisometric on the ideal $J_{\mathscr{R}(\mathscr{E})}$ with $\sigma_{p}^{\#}\left(J_{\mathscr{R}(\mathscr{E})}\right) \subseteq J_{\mathscr{R}(\mathscr{E} 1)}$.

Proof. First note that $J(\mathscr{R}(\mathscr{E}))=C_{0}\left(X_{\text {fin }} \cap r(E)\right), J_{\mathscr{R}(\mathscr{E})}=C_{0}\left(X_{\text {fin }} \cap\right.$ $r(E) \cap \operatorname{Int}(\overline{s(E)}), J(\mathscr{R}(\mathscr{E} 1))=C_{0}\left(X_{\text {fin }}^{1} \cap r_{1}\left(E^{1}\right)\right)=C_{0}\left(X_{\text {fin }}^{1} \cap\left(s^{-1}(r(E)) \sqcup\right.\right.$ $\bar{F}))$ and $J_{\mathscr{R}(\mathscr{E} 1)}=C_{0}\left(X_{\text {fin }}^{1} \cap r_{1}\left(E^{1}\right) \cap \operatorname{Int}\left(\overline{s_{1}\left(E^{1}\right)}\right)\right)$. In general $s_{1}\left(E^{1}\right)$ is dense in $E$, so $r_{1}\left(E^{1}\right) \cap \operatorname{Int}\left(\overline{s_{1}\left(E^{1}\right)}\right)=s^{-1}(r(E)) \subseteq E$ and therefore $J_{\mathscr{R}(\mathscr{E} 1)}=$ $C_{0}\left(X_{\text {fin }}^{1} \cap s^{-1}(r(E))\right)$.

View the map $\left(\phi_{1}\right)_{I_{1}}(a)$ on $\left.\mathscr{R}(\mathscr{E})^{1}\right)$ as $M_{j^{*} a} \otimes \operatorname{Id}_{I_{1}}$ on $\mathscr{I}_{I_{1}} \mathscr{E}_{I} \otimes_{I}\left(I_{1}\right)_{I_{1}}$ for $a \in C_{0}\left(s^{-1}(r(E)) \oplus C_{0}(\bar{F})=I_{1}\right.$ where $M$ is the multiplication representation of $C_{b}(E)$ in $\mathscr{L}(\mathscr{E})$ and $j$ is the inclusion of $s^{-1}(r(E))$ into $s^{-1}(r(E)) \sqcup \bar{F}$ (comments following Theorem 2.9). Remark 3.13 of [14] shows that $M^{-1}(\mathscr{K}(\mathscr{E}))=$ $C_{0}(U)$ where $U$ is the largest open set of $E$ with the property that $\left.s\right|_{U}$ is a proper map and $\left.r\right|_{U}$ is a local homeomorphism. In particular the description of $X_{\text {fin }}$ implies that $s^{-1}\left(X_{\text {fin }}\right) \subseteq U$, so the action $M$ of $C_{0}\left(s^{-1}\left(X_{\text {fin }}\right)\right)$ is by elements in $\mathscr{K}(\mathscr{E})$. Since $j^{\#}(a)=0$ for $a \in C_{0}(\bar{F})$ we have that $M_{j^{*} a} \in \mathscr{K}(\mathscr{E})$ for $a \in C_{0}\left(s^{-1}\left(X_{\text {fin }} \cap r(E)\right)\right) \oplus C_{0}(\bar{F})$ and so (cf. the first paragraph of Proposition 3.7) $\left(\phi_{1}\right)_{I_{1}}(a)=M_{j^{\#} a} \otimes \operatorname{Id}_{I_{1}} \in \mathscr{K}(\mathscr{E} 1)$ for $a \in$ $C_{0}\left(s^{-1}\left(X_{\text {fin }} \cap r(E)\right)\right) \oplus C_{0}(\bar{F})$. Thus $C_{0}\left(s^{-1}\left(X_{\text {fin }} \cap r(E)\right)\right) \oplus C_{0}(\bar{F})$ is contained in $J(\mathscr{R}(\mathscr{E} 1))$, so $s^{-1}\left(X_{\text {fin }} \cap r(E) \cap \operatorname{Int}(\overline{s(E)})\right) \sqcup \bar{F}$ is contained in $X_{\text {fin }}^{1} \cap\left(s^{-1}(r(E)) \sqcup \bar{F}\right)$ and $s^{-1}\left(X_{\text {fin }} \cap r(E) \cap \operatorname{Int}(\overline{s(E)})\right)$ is contained in $X_{\text {fin }}^{1} \cap s^{-1}(r(E))$. By Proposition 2.7 and the comments following we see that the later containment shows $C_{0}\left(s^{-1}\left(X_{\mathrm{fin}} \cap r(E) \cap \operatorname{Int}(\overline{s(E)})\right)\right)$ is contained in $J_{\mathscr{R}(\mathscr{E} 1)}$. Since $\sigma_{p}^{\#}(J(\mathscr{R}(\mathscr{E}))) \subseteq C_{0}\left(s^{-1}\left(X_{\text {fin }} \cap r(E)\right)\right) \oplus C_{0}(\bar{F})$ and $\sigma_{p}^{\#}\left(J_{\mathscr{R}(\mathscr{E})}\right) \subseteq$ $C_{0}\left(s^{-1}\left(X_{\text {fin }} \cap r(E) \cap \operatorname{Int}(\overline{s(E)})\right)\right)$ we have $\sigma_{p}^{\#}(J(\mathscr{R}(\mathscr{E}))) \subseteq J(\mathscr{R}(\mathscr{E} 1))$ and $\sigma_{p}^{\#}\left(J_{\mathscr{R}(\mathscr{E})}\right) \subseteq J_{\mathscr{R}(\mathscr{E} 1)}$

For $a \in J_{\mathscr{R}(\mathscr{E})}=C_{0}\left(X_{\text {fin }} \cap r(E) \cap \operatorname{Int}(\overline{s(E)})\right)$ we need to show that $\Psi_{\left(s_{1}\right)^{\#}}\left(\phi_{I}(a)\right)=\phi_{I_{1}}\left(\sigma_{p}^{\#}(a)\right)$. The comments after Theorem 2.9 show that 
$\left(\phi_{1}\right)_{I_{1}}(a)=M_{j^{*} a} \otimes \operatorname{Id}_{I_{1}}$ for $a \in C_{0}\left(s^{-1}(r(E)) \oplus C_{0}(\bar{F})=I_{1}\right.$. However, for $a \in C_{0}\left(X_{\mathrm{fin}} \cap r(E) \cap \operatorname{Int}(\overline{s(E)})\right)$ we have that the element $\sigma_{p}^{\#}(a)$ of $C_{0}\left(s^{-1}(r(E))\right) \oplus C_{0}(\bar{F})$ is equal to the element $\left(s_{p}^{\#}(a), 0\right)$, so $j^{\#}\left(\sigma_{p}^{\#}(a)\right)=$ $s_{p}^{\#}(a) \in C_{0}\left(s^{-1}\left(X_{\text {fin }} \cap r(E) \cap \operatorname{Int}(\overline{s(E)})\right)\right)$. Thus $\left(\phi_{1}\right)_{I_{1}}\left(\sigma_{p}^{\#}(a)\right)=M_{s_{p}^{\#}(a)} \otimes \operatorname{Id}_{I_{1}}$. Now $M_{s_{p}^{\#}(a)}=\phi_{I}(a)$ on $\mathscr{R}(\mathscr{E})$ and Proposition 3.7 shows $\Psi_{\left(s_{1}\right)^{*}}\left(\phi_{I}(a)\right)=$ $\phi_{I}(a) \otimes \operatorname{Id}_{I_{1}}$.

Corollary 3.9. If $G$ is a proper topological quiver, $G^{1}$ the associated topological relation and $\mathscr{E}=\mathscr{E}_{G}, \mathscr{E} 1=\mathscr{E}_{G^{1}}$ then there is an injective $*_{-}$ homomorphism $\tau: \mathscr{O}_{\mathscr{R}(\mathscr{E})} \rightarrow \mathscr{O}_{\mathscr{R}(\mathscr{E} 1)}$

Proof. The first paragraph of Proposition 3.6 shows that $\sigma_{p}^{\#}$ is injective while the previous theorem shows that the morphism $\left(s_{1}^{\#}, \sigma_{p}^{\#}\right): \mathscr{R}(\mathscr{E}) \rightarrow$ $\left.\mathscr{R}(\mathscr{E})^{1}\right)$ satisfies the conditions of Corollary 1.5.

\section{Range finite topological quivers}

We introduce conditions on a topological quiver to ensure that the unaugmented Cuntz-Pimsner $\mathrm{C}^{*}$-algebras of a topological quiver and its associated relation are isomorphic. One of these conditions involves the left action acting by compact elements on the correspondence. Another condition is that the kernel of the restricted left action $\operatorname{ker}\left(\phi_{I}\right)$ is complemented in $\overline{\langle\mathscr{E}, \mathscr{E}\rangle}$.

Definition 4.1. A topological quiver $G=(X, E, r, s, \lambda)$ is range finite if $r(E) \subseteq X_{\text {fin }}$.

Lemma 4.2. A topological quiver $G=(X, E, r, s, \lambda)$ is range finite if and only if $\operatorname{Im}\left(\phi_{I}\right) \subseteq \mathscr{K}(\mathscr{E})$ for the associated restricted correspondence $\mathscr{R}(\mathscr{E})$.

Proof. Since $\phi^{-1}(\mathscr{K}(\mathscr{E}))=C_{0}\left(X_{\text {fin }}\right)$ where $\phi$ is the left action for $\mathscr{E}$ and $\phi_{I}$ is defined on $C_{0}(r(E))$ we have $\operatorname{Im}\left(\phi_{I}\right) \subseteq \mathscr{K}(\mathscr{E})$ if and only if $r(E) \subseteq X_{\text {fin }}$.

Since $X_{\text {fin }} \subseteq X_{p}(s)$ a range finite topological quiver $G$ must necessarily be proper. As is the case with $X_{p}, X_{\text {fin }}$ always contains $X-\overline{s(E)}$, and so

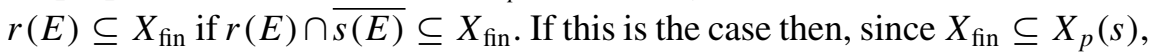
Lemma 3.2 implies $r(E) \cap \overline{s(E)}=r(E) \cap s(E)$. Since $X_{\text {fin }}=X_{p}(s)$ whenever $r$ restricted to $s^{-1}(r(E))$ is a local homeomorphism we have that $G$ is proper if and only if $G$ is range finite for such $G$. So, for example, if $G$ is a discrete directed graph, or arises from a continuous map $f: X \rightarrow Y$, or is a topological graph in the sense of Katsura ([8]), then there is no distinction between proper and range finite.

Proposition 4.3. If $G$ is a range finite quiver then the associated topological relation $G^{1}$ is range finite. 
Proof. By the above comment it is enough to show that $r_{1}\left(E^{1}\right) \cap\left(\overline{s_{1}\left(E^{1}\right)}\right)=$ $s^{-1}(r(E))$ is contained in $\left(X^{1}\right)_{\text {fin }}$. By the previous theorem $C_{0}\left(s^{-1}\left(X_{\text {fin }} \cap\right.\right.$ $r(E))) \oplus C_{0}(\bar{F})$ is contained in $J\left(\mathscr{R}\left(\mathscr{E}^{1}\right)\right)$ and since $r(E) \subseteq X_{\text {fin }}$ we have $C_{0}\left(s^{-1}(r(E))\right)$ is contained in $J\left(\mathscr{R}\left(\mathscr{E}^{1}\right)\right)=C_{0}\left(\left(X^{1}\right)_{\text {fin }} \cap r_{1}\left(E^{1}\right)\right)$.

A final condition that will ensure that the unaugmented Cuntz-Pimsner $\mathrm{C}^{*-}$ algebras of a topological quiver $G$ and its desingularization are isomorphic involves a topological condition on the quiver $G$; namely that the set of nonisolated sinks $F=r(E)-\overline{s(E)}$ is closed, hence clopen, in $r(E)$. That $F$ is clopen in $r(E)$ is certainly the case when $F$ is empty or when the subspace $r(E)$ of the vertex space $X$ is discrete, which, since $r$ is an open map, entails conditions on $E$ also. Note that this condition is automatically satisfied for the desingularized quiver $G^{1}$ as we have (Proposition 2.7) that $F_{G^{1}}=r_{1}\left(E^{1}\right)-\overline{s_{1}\left(E^{1}\right)}=\bar{F}$, a clopen set in $r_{1}\left(E^{1}\right)$.

In the following definition the condition that $F$ is clopen implies that $C_{0}(\bar{F})$, which is a direct summand in the $\mathrm{C}^{*}$-algebra $I_{1}=C_{0}\left(r_{1}\left(E^{1}\right)\right)$, is the same as $C_{0}(F)$, the ideal $\operatorname{ker}\left(\phi_{I}\right)$ in the $\mathrm{C}^{*}$-algebra $I=C_{0}(r(E))$. Thus if $\pi$ is a representation of $I$ we may apply it to this direct summand $C_{0}(F)$ of $I_{1}$. Also recall that for $G$ a range finite quiver the map $\left(\phi_{1}\right)_{I_{1}}(b)$ on $\mathscr{R}(\mathscr{E} 1)$ is the map $M_{j^{*} b} \otimes \operatorname{Id}_{I_{1}}$ where $M_{j^{*} b} \in \mathscr{K}(\mathscr{E})$ for $b \in I_{1}=C_{0}\left(s^{-1}(r(E))\right) \oplus C_{0}(F)$ (Theorem 3.8) and $j$ is the inclusion $s^{-1}(r(E))$ into $s^{-1}(r(E)) \sqcup F$. Writing an element $b$ of $I_{1}$ as $\left(b_{1}, b_{2}\right)$ we have $j^{\#} b=b_{1}$.

Definition 4.4. Let $G=(X, E, r, s, \lambda)$ be a range finite topological quiver with $F_{G}$ closed in $r(E)$. For $(T, \pi): \mathscr{R}\left(\mathscr{E}_{G}\right) \rightarrow B$ a representation of the correspondence $\mathscr{R}\left(\mathscr{E}_{G}\right)$ in a $\mathrm{C}^{*}$-algebra $B$ define $\left(T_{1}, \pi_{1}\right): \mathscr{R}\left(\mathscr{E}^{1}\right) \rightarrow B$ as follows: for $b=\left(b_{1}, b_{2}\right) \in I_{1}=C_{0}\left(s^{-1}(r(E))\right) \oplus C_{0}(F)$ set $\pi_{1}(b)=$ $\Psi_{T}\left(M_{b_{1}}\right)+\pi\left(b_{2}\right)$, for $g \otimes h \in C_{c}(E) \otimes C_{c}\left(s^{-1}(r(E)) \sqcup F\right)$ set $T_{1}(g \otimes h)=$ $T(g) \pi_{1}(h)$.

Using Proposition 1.2 we have for $c \in C_{0}\left(s^{-1}(r(E))\right)$ and $d \in C_{0}(F)=$ $\operatorname{ker} \phi_{I}$ that $\pi(d) \Psi_{T}\left(M_{c}\right)=\Psi_{T}\left(\phi_{I}(d) M_{c}\right)=0$ and also $\Psi_{T}\left(M_{c}\right) \pi(d)=0$. It follows that $\pi_{1}$ is a $*$-homomorphism of $\mathrm{C}^{*}$-algebras. In fact more is true; the pair $\left(\pi_{1}, \pi\right)$ may be viewed as a representation of the correspondence ${ }_{I}\left(I_{1}\right)_{I_{1}}$ in the $\mathrm{C}^{*}$-algebra $B$ where we recall the definition of the left action of $I$ on $I_{1}$ is via the map $\sigma^{\#}$. To see this we need to check $\pi(a) \pi_{1}(b)=\pi_{1}(a b)$ for $a \in$ $I=C_{0}(r(E))$ and $b \in I_{1}$. The left side $\pi(a) \pi_{1}\left(b_{1}, b_{2}\right)=\pi(a)\left[\Psi_{T}\left(M_{b_{1}}\right)+\right.$ $\left.\pi\left(b_{2}\right)\right]$, which by Proposition 1.2 is equal to $\Psi_{T}\left(\phi_{I}(a) M_{b_{1}}\right)+\pi\left(a b_{2}\right)=$ $\Psi_{T}\left(M_{\sigma_{p}^{\#}(a) b_{1}}\right)+\pi\left(a b_{2}\right)=\Psi_{T}\left(M_{s^{\#}(a) b_{1}}\right)+\pi\left(a b_{2}\right)=\pi_{1}\left(\left(s^{\#}(a) b_{1}, a b_{2}\right)\right)$. This is the right side by the definition of the left action of $I$ on $I_{1}$.

Clearly $T_{1}$ defines a linear map on the algebraic tensor product. For $a \in I$ we have $T_{1}(g a \otimes h)=T(g a) \pi_{1}(h)=T(g) \pi(a) \pi_{1}(h)=T(g) \pi_{1}(a h)=$ 
$T_{1}(g \otimes a h)$. Thus $T_{1}$ is a well defined linear map from the pre-Hilbert $I_{1^{-}}$ module interior algebraic tensor product $C_{c}(E)_{I} \otimes_{\text {alg } I} C_{c}\left(r_{1}\left(E^{1}\right)\right)$ to $B$.

Proposition 4.5. Let $G$ be a range finite topological quiver with $F$ closed in $r(E)$ and $(T, \pi): \mathscr{R}\left(\mathscr{E}_{G}\right) \rightarrow B$ a representation in a $C^{*}$-algebra $B$. The map $\left(T_{1}, \pi_{1}\right): \mathscr{R}(\mathscr{E} 1) \rightarrow B$ defined above is a representation of the correspondence $\mathscr{R}\left(\mathscr{E}^{1}\right)$. If $(T, \pi)$ admits a gauge action then so does $\left(T_{1}, \pi_{1}\right)$. If $\pi$ is injective then so is $\pi_{1}$.

Proof. For $g \otimes h$ and $k \otimes l \in C_{c}(E)_{I} \otimes_{\text {alg }} C_{c}\left(r_{1}\left(E^{1}\right)\right)$ we first show that $T_{1}(g \otimes h)^{*} T_{1}(k \otimes l)=\pi_{1}\left(\langle g \otimes h, k \otimes l\rangle_{I_{1}}\right)$. The left side is $\pi_{1}(\bar{h}) T(g)^{*} T(k) \pi_{1}(l)$ $=\pi_{1}(\bar{h}) \pi\langle g, k\rangle_{I} \pi_{1}(l)=\pi_{1}(\bar{h}) \pi_{1}\left(\langle g, k\rangle_{I}(l)\right)=\pi_{1}\left(\bar{h}\left(\langle g, k\rangle_{I} l\right)\right)$ which is the right side. Thus $T_{1}$ is continuous (bounded by 1 ) and extends to a linear map on the completed tensor product correspondence $\mathscr{R}(\mathscr{E} 1)$.

For $b \in I_{1}$ and $g \in C_{c}(E)$ we compute, using Proposition 1.2, that $\pi_{1}(b) T(g)$ $=\left[\Psi_{T}\left(M_{b_{1}}\right)+\pi\left(b_{2}\right)\right] T(g)=T\left(M_{b_{1}}(g)\right)+T\left(\phi_{I}\left(b_{2}\right) g\right)=T\left(M_{b_{1}}(g)\right)$ since $b_{2} \in \operatorname{ker}\left(\phi_{I}\right)$. We check the second condition of Definition 1.1:

$$
\begin{aligned}
T_{1}\left(\left(\phi_{1}\right)_{I_{1}}(b)(g \otimes h)\right) & =T_{1}\left(M_{b_{1}} \otimes \operatorname{Id}_{I_{1}}\right)(g \otimes h)=T_{1}\left(b_{1} g \otimes h\right) \\
& =T\left(b_{1} g\right) \pi_{1}(h)=\pi_{1}(b) T(g) \pi_{1}(h)=\pi_{1}(b) T_{1}(g \otimes h) .
\end{aligned}
$$

If $\gamma: \mathrm{T} \rightarrow$ Aut $C^{*}(T, \pi)$ is a gauge action then $\gamma_{t}\left(\pi_{1}(b)\right)=\gamma_{t} \Psi_{T}\left(M_{b_{1}}\right)+$ $\gamma_{t} \pi\left(b_{2}\right)=\pi_{1}(b)$ and $\gamma_{t} T_{1}(g \otimes h)=\gamma_{t}\left(T(g) \pi_{1}(h)\right)=\gamma_{t}(T(g)) \pi_{1}(h)=$ $t T_{1}(g \otimes h)$ so $\gamma$ restricts to an element of Aut $C^{*}\left(T_{1}, \pi_{1}\right)$ and is a gauge action for $\left(T_{1}, \pi_{1}\right)$.

If $\pi$ is injective on $I$ then $T$ is an isometry on $C_{c}(E)$. Suppose $\pi_{1}(b)=0$ for some $b \in I_{1}$. Then $0=\pi_{1}(b) T(g)=T\left(b_{1} g\right)$ for all $g \in C_{c}\left(s^{-1}(r(E))\right)$ and since $T$ is injective $b_{1} g=0$ for all such $g$, where $b_{1}$ is an element of $C_{0}\left(s^{-1}(r(E))\right)$. Thus $b_{1}=0$ and $0=\pi_{1}(b)=\pi\left(b_{2}\right)$, and since $\pi$ is injective $b_{2}$, and thus also $b=0$.

THEOREM 4.6. Let $G$ be a range finite topological quiver with $F_{G}$ closed in $r(E), \mathscr{E}=\mathscr{E}_{G}$, and $(T, \pi): \mathscr{R}\left(\mathscr{E}_{G}\right) \rightarrow B$ a representation in a $C^{*}$-algebra $B$ coisometric on $J_{\mathscr{E}}$. Then $(T, \pi)=\left(T_{1}, \pi_{1}\right) \circ\left(s_{1}^{\#}, \sigma_{p}^{\#}\right)$.

Proof. We first show $\pi_{1} \circ \sigma_{p}^{\#}=\pi$ on $I=C_{0}(r(E) \cap s(E)) \oplus C_{0}(F)$. Writing $k=\left(k_{1}, k_{2}\right) \in I$ we have $\pi_{1}\left(\sigma_{p}^{\#}(k)\right)=\pi_{1}\left(\left(s^{\#} k_{1}, k_{2}\right)\right)=\Psi_{T} \phi_{I}\left(k_{1}\right)+$ $\pi\left(k_{2}\right)$. Using that $(T, \pi)$ is coisometric on $J_{\mathscr{E}}=C_{0}(r(E) \cap s(E))$ this is $\pi\left(k_{1}\right)+\pi\left(k_{2}\right)=\pi(k)$.

To show $T=T_{1} \circ s_{1}^{\#}$ on $\mathscr{E}$ it is enough to show this on the dense subspace $C_{c}(E)$. For $g \in C_{c}(E)$ choose a real valued element $\left.k \in C_{c}(r(E))\right)$ with values between 0 and 1 so that $k=1$ on the compact set $r(\operatorname{supp}(g))$. Then $\sigma_{p}^{\#}(k) \in C_{c}\left(s^{-1}(r(E)) \sqcup F\right)$ and $g \otimes \sigma_{p}^{\#}(k)=s_{1}^{\#}(g)$ as elements of $C_{c}\left(E^{1}\right)$ and 
$\left(T_{1} \circ s_{1}^{\#}\right)(g)=T_{1}\left(g \otimes \sigma_{p}^{\#}(k)\right)=T(g) \pi_{1}\left(\sigma_{p}^{\#}(k)\right)=T(g) \pi(k)=T\left(g\left(r^{\#}(k)\right)=\right.$ $T(g)$ by the choice of $k$.

COROLlaRY 4.7. With the hypothesis of the previous theorem the representation $\left(T_{1}, \pi_{1}\right): \mathscr{R}\left(\mathscr{E}^{1}\right) \rightarrow B$ is coisometric on $J_{\mathscr{E} 1}$.

Proof. For $b=(b, 0)$ an element of the ideal $J_{\mathscr{E} 1}=C_{0}\left(s^{-1}(r(E))\right)$ of $I_{1}=$ $C_{0}\left(s^{-1}(r(E))\right) \oplus C_{0}(F)$ (Proposition 2.7), $\Psi_{T_{1}}\left(\left(\phi_{1}\right)_{I_{1}}(b)\right)=\Psi_{T_{1}}\left(M_{b} \otimes \operatorname{Id}_{I_{1}}\right)=$ $\Psi_{T_{1}}\left[\Psi_{s_{1}^{\#}}\left(M_{b}\right)\right]$ by Proposition 3.7. This however equals $\Psi_{T_{1} \circ s_{1}^{*}}\left(M_{b}\right)=\Psi_{T}\left(M_{b}\right)$ by the above theorem, and this is $\pi_{1}(b)$.

THEOREM 4.8. Let $G$ be a range finite topological quiver with $F_{G}$ closed in $r(E)$ and $G^{1}$ the associated topological relation. With $\mathscr{E}=\mathscr{E}_{G}$ and $\mathscr{E} 1=\mathscr{E}_{G^{1}}$ then $\mathscr{O}_{\mathscr{R}(\mathscr{E})} \cong \mathscr{O}_{\mathscr{R}(\mathscr{E} 1)}$ via the *-homomorphism $\tau$ of Corollary 3.9; so the unaugmented Cuntz-Pimsner $C^{*}$-algebras of the topological quiver $G$ and its desingularization are isomorphic.

Proof. For $(T, \pi): \mathscr{R}(\mathscr{E}) \rightarrow \mathscr{O}_{\mathscr{R}(\mathscr{E})}$ a universal representation coisometric on $I=J_{\mathscr{E}}$ there is a representation $\left(T_{1}, \pi_{1}\right): \mathscr{R}(\mathscr{E} 1) \rightarrow \mathscr{O}_{\mathscr{R}(\mathscr{E})}$ coisometric on $I_{1}=J_{\mathscr{E} 1}$. Since $(T, \pi)$ admits a gauge action, so does $\left(T_{1}, \pi_{1}\right)$, and since $\pi$ is injective then so is $\pi_{1}$. Thus the *-homomorphism $\rho: \mathscr{O}_{\mathscr{R}(\mathscr{E} 1)} \rightarrow \mathscr{O}_{\mathscr{R}(\mathscr{E})}$ ensured by the universal property applied to $\left(T_{1}, \pi_{1}\right)$ is injective by the gauge invariant uniqueness theorem. By considering the diagram of morphisms and *-homomorphisms we have that $\rho \circ \tau \circ(T, \pi)=\left(T_{1}, \pi_{1}\right) \circ\left(s_{1}^{\#}, s^{\#}\right)$, which by Theorem 4.6 is $(T, \pi)$. Therefore the *-homomorphism $\rho \circ \sigma$ must be the identity map on the generators $\operatorname{Im} T(\mathscr{E})$ and $\pi(I)$ of $\mathscr{O}_{\mathscr{R}(\mathscr{E})}$, so $\rho \circ \tau=\operatorname{Id}_{\left.\mathscr{O}_{\mathscr{R}} \mathscr{E}\right)}$. Thus $\rho$ is surjective as well as injective, and both $\rho$ and $\tau$ are *-isomorphisms.

Corollary 4.9. Let $G$ be a range finite topological quiver and $G^{1}$ the associated topological relation. If $\mathscr{E}^{1}=\mathscr{E}_{G^{1}}$ and $\mathscr{E}^{1,1}=\mathscr{E}_{\left(G^{1}\right)^{1}}$ then $\mathcal{O}_{\mathscr{R}(\mathscr{E} 1)} \cong$ $\mathcal{O}_{\mathscr{R}(\mathscr{E} 1,1)}$.

Proof. We have noted that $F_{G^{1}}$ is clopen for the desingularized quiver $G^{1}$. Proposition 4.3 then shows that $G^{1}$ satisfies the hypothesis of the theorem.

We conclude with some examples. First note that if $G$ is a discrete graph then $G$ is range finite if and only if $G$ is proper, while $F_{G}$ is automatically closed in $r(E)$, so the hypothesis of the previous theorem are immediately satisfied for proper discrete graphs. We illustrate that the $\mathrm{C}^{*}$-algebras $\mathscr{O}_{\mathscr{R}(\mathscr{E})}$ and $\mathscr{O}_{\mathscr{R}(\mathscr{E} 1)}$ are generally not isomorphic even for discrete directed graphs $G$ which are not proper. Consider the non-proper discrete directed graph with two vertices $p, q$, and with edges $u$ from $q$ to $p$, and $w_{i}$ from $p$ to $q(i \in \mathrm{N})$. This graph has no sources or sinks, and its associated discrete directed graph $G^{1}$ is a graph with an infinite (discrete) vertex space with vertices $u, w_{k},(k \in \mathrm{N})$ 
and an edge from $u$ to each $w_{k}$ and also from each $w_{k}$ to $u$. Since the vertex space of $G$ is compact and that of $G^{1}$ is not, the $\mathrm{C}^{*}$-algebra $\mathscr{O}_{\mathscr{R}(\mathscr{E})}$ is unital while the $\mathrm{C}^{*}$-algebra $\mathscr{O}_{\mathscr{R}(\mathscr{E} 1)}$ is nonunital ([14]). The discrete directed graph with an infinite number of loops on a single vertex yielding the Cuntz algebra $\mathcal{O}_{\infty}$ furnishes a similar example.

We next show that the failure of either condition in the last theorem can lead to a failure of the conclusion. The first illustrates non-isomorphic, and non Morita equivalent, algebras $\mathcal{O}_{\mathscr{R}(\mathscr{E})}$ and $\mathscr{O}_{\mathscr{R}(\mathscr{E} 1)}$ where the left action $\phi_{I}$ is not by elements in $\mathscr{K}(\mathscr{E})$ but $F_{G}$ closed in $r(E)$ (since it is empty).

Let $G=(X, E, r, s, \lambda)$ be the proper topological quiver given by $X=\{v\}$ a point, $E=\mathrm{T}$, and $r(t)=s(t)=v$ for all $t \in \mathrm{T}$ with $\lambda$ normalized Haar measure $\mu$ on T. One may view this as a loop of multiplicity T on a single vertex $v$. Since $r(E)=X$ the restricted correspondence $\mathscr{R}\left(\mathscr{E}_{G}\right)=\mathscr{E}_{G}$ is the Hilbert space $L^{2}(\mathrm{~T}, \mu)$ over $\mathrm{C}=C(\{v\})$ with left action $\phi(a)$ given by multiplication $M_{a}$ by the scalar $a \in \mathrm{C}$ on $L^{2}(\mathrm{~T}, \mu)$. Thus $\operatorname{ker}\left(\phi_{I}\right)$ is zero and $F_{G}$ is empty. Also $\phi^{-1}(\mathscr{K}(\mathscr{E}))$ is the zero ideal in C, and $J(\mathscr{E})=J_{\mathscr{E}}=0$. If $(T, \pi)$ is a representation of $\mathscr{E}$ then $T$ is a linear isometry and is determined by its values $T_{n}=T\left(t^{n}\right)$ on the orthogonal elements $t^{n}$ of $L^{2}(\mathrm{~T}, \mu), n \in \mathrm{Z}$. Condition 2 of 1.1 ensures that $T_{n}^{*} T_{m}=\delta_{n, m}$ so the universal $\mathrm{C}^{*}$-algebra $\mathscr{O}_{\mathscr{E}}$ is the Cuntz algebra $\mathscr{O}_{\infty}$, with $K_{0}$ group $\mathrm{Z}$ and trivial $K_{1}$ group.

The associated topological relation $G^{1}=\left(X^{1}, E^{1}, r_{1}, s_{1}, \lambda^{1}\right)$ has $X^{1}=\mathrm{T}$, $E^{1}=\mathrm{T} \times \mathrm{T}, s_{1}$ and $r_{1}$ the projections of $\mathrm{T} \times \mathrm{T}$ onto the first and second components respectively, and $\lambda_{t}^{1}=\mu$ for $t \in \mathrm{T}$. The correspondence $\mathscr{R}(\mathscr{E} 1)=$ $\mathscr{E} 1$ is the correspondence described in example c) of [2] where it is shown that the $\mathrm{C}^{*}$-algebra $\mathcal{O}_{\mathscr{E} 1}$ is isomorphic to the crossed product of the Cuntz algebra $\mathcal{O}_{\infty}$ by an outer automorphism. It is a classifiable unital Kirchberg algebra with both $K$-groups isomorphic to Z. Thus $\mathscr{O}_{\mathscr{R}(\mathscr{E})}$ is not Morita equivalent to $\mathscr{O}_{\mathscr{R}(\mathscr{E} 1)}$.

We next describe an example of a range finite quiver $G$, in fact a topological relation arising from a function, where $F_{G}$ is not closed in $r(E)$ and the associated $\mathrm{C}^{*}$-algebras for $G$ and $G^{1}$ are neither isomorphic nor Morita equivalent.

For $v \in \mathrm{T}$ define a topological quiver $G=(X, E, r, s, \lambda)$ with $X=\mathrm{T}$, $E \cong \mathrm{T}$ with elements $\left\{e_{t} \mid t \in \mathrm{T}\right\}, r\left(e_{t}\right)=t, s\left(e_{t}\right)=v$ and $\lambda_{t}$ counting measure on $r^{-1}(t)=\left\{e_{t}\right\}$. This describes the topological relation arising from the function on T with constant value $v$ ([2]). Since $r(E)=X$ the restricted correspondence $\mathscr{R}\left(\mathscr{E}_{G}\right)$ is identical with the correspondence $\mathscr{E}_{G}$ where $\mathscr{E}_{G}=\mathscr{E}$ is the standard Hilbert module of the $\mathrm{C}^{*}$-algebra $C(\mathrm{~T})$ over itself, with the left action $\phi(f)$ equal to multiplication by the scalar value $f(v), f \in C(\mathrm{~T})$. Since $C(\mathrm{~T})$ is unital this left action is by elements of $\mathscr{K}(\mathscr{E})$, so $r(E) \subseteq X_{\text {fin }}$ and the quiver is range finite. Also $\operatorname{ker} \phi$ is the ideal $C_{0}(F)$ with $F=\mathrm{T}-\{v\}$. Thus $\bar{F}=\mathrm{T}$ and the ideal $J_{\mathscr{E}}=\phi^{-1}(\mathscr{K}(\mathscr{E})) \cap(\operatorname{ker} \phi)^{\perp}=C(\mathrm{~T}) \cap C_{0}(F)^{\perp}=0$. 
The unaugmented Cuntz-Pimsner $\mathrm{C}^{*}$-algebra $\mathcal{O}_{\mathscr{R}(\mathscr{E})}$ is identical to the $\mathrm{C}^{*}$ algebra $\mathscr{O}_{\mathscr{E}}$ which, since $J_{\mathscr{E}}=0$, is the universal Toeplitz $\mathrm{C}^{*}$-algebra $\mathscr{T}(\mathscr{E})$. If $(T, \pi)$ is a representation of $\mathscr{E}$ in a $C^{*}$-algebra $B$, condition 2 of Definition 1.1 shows $T(1)$ is a partial isometry with initial projection $\pi(1)$ where 1 is the unit of $C(\mathrm{~T})$. If $p$ is the final projection of $T(1)$ we have from condition 3 that $\pi(1) p=1(v) p=1 p=p$, so $p \leq \pi(1)$. Thus $\pi(1) T=\pi(1) T T^{*} T=$ $\pi(1) p T=p T=T$ and it follows that $\pi(1)$ is the unit for $C^{*}(T, \pi)$. So $T(1)$ is an isometry, and $\mathscr{T}(\mathscr{E})$ is the universal $\mathrm{C}^{*}$-algebra generated by an isometry $T$ and the $\mathrm{C}^{*}$-algebra $C(\mathrm{~T})$ with $\pi(f) T=f(v) T$. One can view this as adjoining both the spectral projection for the point $\{v\}$ of $\mathrm{T}$ as well as an isometry $T$ with this projection as its final projection to the algebra $C(\mathrm{~T})$. By Propositions 2.7 and 2.8 of [9] there is a *homomorphism of $\mathscr{T}(\mathscr{E})$ to the Toeplitz algebra $\mathscr{T}$ with kernel $C_{0}(\mathrm{~T}-\{v\}) \otimes \mathscr{K}$ and the $K$-groups of $\mathscr{T}(\mathscr{E})$ are isomorphic to the $\mathrm{K}$-groups of $C(\mathrm{~T})$; so both $K$-groups are isomorphic to the group $Z$.

The topological relation $G^{1}=\left(X^{1}, E^{1}, r_{1}, s_{1}, \lambda^{1}\right)$ associated with $G$ has $X^{1}=E \sqcup \mathrm{T}$ while $E^{1}$ is homeomorphic to $X^{1}$ via the map $r_{1}$, and $F_{G^{1}}=$ $\bar{F}=\mathrm{T}$. Again, the restricted correspondence $\mathscr{R}\left(\mathscr{E}^{1}\right)$ is identical with the correspondence $\mathscr{E} 1$, so $\mathscr{O}_{\mathscr{R}(\mathscr{E} 1)}$ is $\mathscr{O}_{\mathscr{E} 1}$, and $\mathscr{E} 1$ is the standard Hilbert module of the unital $\mathrm{C}^{*}$-algebra $C\left(E^{1}\right)=C(\mathrm{~T}) \oplus C(\mathrm{~T})$ over itself. The left action $\phi_{1}(a, b)$ is given by $M_{a\left(e_{v}\right)} \oplus M_{a}$ for $(a, b) \in C\left(E^{1}\right)$, where $M$ is the left multiplication representation. This topological relation still arises from a function (defined on $X^{1}$ ) but it is no longer a constant function. The ideal $J_{\mathscr{E} 1}=C(E) \oplus 0$ and the algebra $\mathscr{O}_{\mathscr{E} 1}$ is the universal $\mathrm{C}^{*}$-algebra for representations coisometric on this ideal. Just as in the previous paragraph if $(T, \pi)$ is a representation of $\mathscr{E} 1$ in a $\mathrm{C}^{*}$-algebra $B$ then $T$ is an isometry. For $(a, b) \in C\left(E^{1}\right)$ one can check that the representation must satisfy $\pi(a, b) T=a\left(e_{v}\right) T \pi(1, a)$ and $T T^{*}=\pi(1,0)$, the last arising from the coisometric condition. Set $(T, \pi)$ to be a universal representation of $\mathscr{E}^{1}$ in the $\mathrm{C}^{*}$-algebra $\mathscr{O}_{\mathscr{E}}$.

Consider the hereditary and saturated (Section 8 of [14]) open subset $U=$ $\left(E-\left\{e_{v}\right\}\right) \sqcup(\mathrm{T}-\{v\})$ of $X^{1}$ and form the quiver $G_{U}^{1}=\left(X_{U}^{1}, E_{U}^{1},\left(r_{1}\right)_{U}\right.$, $\left.\left(s_{1}\right)_{U}, \lambda^{U}\right)$ defined in [14], with $X_{U}^{1}=X^{1}-U, E_{U}^{1}=E^{1}-\left(r_{1}\right)^{-1}(U)$, the maps $\left(r_{1}\right)_{U}$ and $\left(s_{1}\right)_{U}$ the restrictions of $r_{1}$ and $s_{1}$ to $U$, and $\lambda^{U}$ the map $\lambda$ restricted to $X_{U}^{1}$. We see that $G_{U}^{1}$ is the directed graph on the two vertices $e_{v}$ and $v$, along with the two edges given by a loop on $e_{v}$ and an edge from $v$ to $e_{v}$. It is known, and easy to check, that the $\mathrm{C}^{*}$-algebra of this directed graph, so the $\mathrm{C}^{*}$-algebra $\mathscr{O}_{\mathscr{E}_{U}}$ where $\mathscr{E}_{U}^{1}$ is the correspondence of the quiver $G_{U}^{1}$, is the usual Toeplitz $\mathrm{C}^{*}$-algebra $\mathscr{T}$ generated by an isometry. The hypothesis of Corollary 8.2.3. of [14] are satisfied, so if $\mathscr{I}_{U}$ denotes the ideal of $\mathscr{O}_{\mathscr{E} 1}$ generated by $\pi\left(C_{0}(U)\right)$ then the quotient $C^{*}$-algebra $\mathscr{O}_{\mathscr{E} 1} /\left(\mathscr{I}_{U}\right)$ is $\mathscr{O}_{\mathscr{E}}$, which we have just seen is $\mathscr{T}$. 
Using the relations defining $\mathscr{O}_{\mathscr{E} 1}$ one can check that the ideal $\mathscr{I}_{U}$ is the closure of $\left\{T^{k}(a, b) T^{* l} \mid k, l \geq 0,(a, b) \in C_{0}(U)\right\}$ and that this is isomorphic to $C_{0}(U) \otimes \mathscr{K}$. We therefore have a short exact sequence $0 \rightarrow C_{0}(U) \otimes$ $\mathscr{K} \rightarrow \mathscr{O}_{\mathscr{R}(\mathscr{E} 1)} \rightarrow \mathscr{T} \rightarrow 0$, and letting $C_{0}(U)^{\sim}$ denote the unitization of $C_{0}(U)$ there is therefore a map of the short exact sequence $0 \rightarrow C_{0}(U) \rightarrow$ $C_{0}(U)^{\sim} \rightarrow \mathrm{C} \rightarrow 0$ to this exact sequence. The same 5-lemma argument used in Proposition 2.8 of [9], where it is noted that the maps $C_{0}(U) \rightarrow C_{0}(U) \otimes \mathscr{K}$ and $\mathrm{C} \rightarrow \mathscr{T}$ are isomorphisms on the level of $K$-theory, show the $K$-groups of $\mathcal{O}_{\mathscr{R}(\mathscr{E} 1)}$ and $C_{0}(U)^{\sim}$ are isomorphic. Thus $K_{0}\left(\mathscr{O}_{\mathscr{R}(\mathscr{E} 1)}\right) \cong K_{0}\left(C_{0}(U)\right) \oplus \mathrm{Z}=\mathrm{Z}$ and $K_{1}\left(\mathcal{O}_{\mathscr{R}(\mathscr{E} 1)}\right) \cong K_{1}\left(C_{0}(U)^{\sim}\right)=\mathrm{K}_{1}\left(C_{0}(U)\right)=\mathrm{Z} \oplus \mathrm{Z}$. Thus $\mathscr{O}_{\mathscr{E}}$ is not isomorphic, nor Morita equivalent, to $\mathscr{O}_{\mathscr{E} 1}$.

ACKNOWLEDGMENT. The author acknowledges support, in connection with this research, from the Natural Sciences and Engineering Research Council of Canada.

\section{REFERENCES}

1. Brenken, B., $C^{*}$-Algebras of infinite graphs and Cuntz-Krieger algebras, Canad. Math. Bull. 45 (2002), 321-336.

2. Brenken, B., $C^{*}$-algebras associated with topological relations, J. Ramanujan Math. Soc. 19 (2004), 35-55.

3. Brenken, B., The isolated ideal of a correspondence associated with a topological quiver, New York J. Math. 12 (2006), 47-62.

4. Brenken, B., A dynamical core for topological directed graphs, Münster J. Math., to appear.

5. Fowler, N., Muhly, P., Raeburn, I., Representations of Cuntz-Pimsner algebras, Indiana Univ. Math. J. 52 (2003), 596-605.

6. Kajiwara, T., Pinzari, C., Watatani, Y., Ideal structure and simplicity of the C*-algebras generated by Hilbert bimodules, J. Funct. Anal. 159 (1998), 295-322.

7. Katsura, T., A construction of $C^{*}$-algebras from $C^{*}$-correspondences, pp. 173-182 in: Advances in Quantum Mechanics, Proc. South Hadley 2002, Contemp. Math. 335, Amer. Math. Soc., Providence, RI 2003.

8. Katsura, T., A class of $C^{*}$-algebras generalizing both graph algebras and homeomorphism $C *$-algebras I: Fundamental results, Trans. Amer. Math. Soc. 356 (2004), 4287-4322.

9. Katsura, T., $C^{*}$-algebras generated by scaling elements, J. Operator Theory 55 (2006), 213222.

10. Katsura, T., A class of $C^{*}$-algebras generalizing both graph algebras and homeomorphism $C^{*}$-algebras II, Internat. J. Math. 17 (2006), 791-833.

11. Lance, E. C., Hilbert $C^{*}$-modules: A Tool Kit for Operator Algebraists, London Math. Soc. Lecture Note Series 210, Cambridge University Press, Cambridge 1995.

12. Muhly, P., Pask, D., Tomforde, M., Strong shift equivalence of $C^{*}$-correspondences, Israel J. Math. 167 (2008), 315-346.

13. Muhly, P., Solel, B., Tensor algebras over $C^{*}$-correspondences: Representations, dilations, and $C^{*}$-envelopes, J. Funct. Anal. 158 (1998), 389-457.

14. Muhly, P., Tomforde, M., Topological quivers, Internat. J. Math. 16 (2005), 693-755. 
15. Pimsner, M. V., A class of $C^{*}$-algebras generalizing both Cuntz-Krieger algebras and crossed products by $Z$, pp. 189-212 in: Free Probability Theory, Proc. Waterloo 1995, Fields Institute Comm. 12, Amer. Math. Soc., Providence, RI 1997.

16. Rørdam, M., Classification of nuclear, simple $C^{*}$-algebras, pp. 1-145 in: Classification of Nuclear $C^{*}$-algebras. Entropy in Operator Algebras, Encyclopaedia Math. Sci. 126, Operator Algebras and Non-commutative Geometry 7, Springer, Berlin 2002.

DEPARTMENT OF MATHEMATICS AND STATISTICS

UNIVERSITY OF CALGARY

2500 UNIVERSITY DRIVE NW

CALGARY, ALBERTA T2N 1N4

CANADA

E-mail: bbrenken@ucalgary.ca 